The Astrophysical Journal, 604:258-271, 2004 March 20

(C) 2004. The American Astronomical Society. All rights reserved. Printed in U.S.A.

\title{
BIPOLAR MOLECULAR OUTFLOWS FROM HIGH-MASS PROTOSTARS
}

\author{
Yu-Nung $\mathrm{Su}^{1}$ \\ Institute of Astronomy, National Central University, Chung-Li 32054, Taiwan; ynsu@asiaa.sinica.edu.tw \\ QizhOU ZHANG \\ Harvard-Smithsonian Center for Astrophysics,60 Garden Street, Cambridge, MA 02138; qzhang@cfa.harvard.edu \\ AND \\ JEREMY LIM \\ Institute of Astronomy and Astrophysics, Academia Sinica, P.O. Box 23-141, Taipei 106, Taiwan; jlim@asiaa.sinica.edu.tw \\ Received 2003 July 30; accepted 2003 November 25
}

\begin{abstract}
We report observations of the bipolar molecular outflows associated with the luminous $\left(\sim 2 \times 10^{4} L \odot\right)$ far-IR sources IRAS $21519+5613$ and IRAS $22506+5944$, as well the dust and molecular gas condensations on which these outflows appear to be centered. The observations were made in ${ }^{12} \mathrm{CO},{ }^{13} \mathrm{CO}, \mathrm{C}^{18} \mathrm{O}$, and continuum at $3 \mathrm{~mm}$ with the BIMA array and in ${ }^{12} \mathrm{CO}$ and ${ }^{13} \mathrm{CO}$ with the NRAO $12 \mathrm{~m}$ telescope to recover extended emission filtered out by the interferometric array. We find that the outflow associated with each IRAS source shows a clear bipolar morphology in ${ }^{12} \mathrm{CO}$, with properties (i.e., total mass of order $10-100 M_{\odot}$, mass-outflow rate $\gtrsim 10^{-3} M_{\odot}$, dynamical timescale $10^{4}-10^{5} \mathrm{yr}$, and energetics) comparable with those of other massive outflows associated with luminous young stellar objects. Each outflow appears to be centered on a dust and gas condensation with a mass of 200-300 $M_{\odot}$, likely marking the location of the driving source. The outflow lobes of both sources are fully resolved along their major but not minor axes, and they have collimation factors that may be comparable with young low-mass stars. The mass-velocity diagrams of both outflows change in slope at a velocity of $\sim 10 \mathrm{~km} \mathrm{~s}^{-1}$, suggesting that the high-velocity component (HVC) may drive the low-velocity component (LVC). Although the HVC of IRAS $21519+5613$ shows evidence for deceleration, no such signature is seen in the HVC of IRAS 22506+5944. Neither HVC has a momentum supply rate sufficient to drive their corresponding LVCs, although it is possible that the HVC is more highly excited and hence its thrust underestimated. Like for other molecular outflows the primary driving agent cannot be ionized gas, leaving atomic gas as the other remaining candidate. Neither IRAS $21519+5613$ nor IRAS $22506+5944$ exhibits detectable free-free emission, which together with the observed properties of their molecular outflows and surrounding condensations make them credible candidates for high-mass protostars. The mass-accretion rate required to produce their observed IRAS luminosity is $\gtrsim 10^{-4} M_{\odot} \mathrm{yr}^{-1}$, which is more than sufficient to quench the development of an $\mathrm{UC}-\mathrm{H}$ II region. On the other hand, the individual IRAS sources may be associated with a group of stars whose dominant member is a main-sequence star that is responsible for the observed outflow. Such a star would be required to have a spectral type of $\sim \mathrm{B} 2$ (luminosity of $\sim 3000 L_{\odot}$ ) or later to not excite a detectable $\mathrm{UC}-\mathrm{H}$ II region; the timeaveraged mass-accretion rate needed to produce this star is then $10^{-3}$ to $10^{-4} M_{\odot} \mathrm{yr}^{-1}$. Thus, regardless of the evolutionary stage of the outflow driving source, the inferred mass-accretion rate is much higher than that allowed by simple inside-out collapse models but can be accommodated by recently proposed variants.
\end{abstract}

Subject headings: ISM: individual (IRAS 21519+5613, IRAS 22506+5944) - ISM: jets and outflows stars: early-type - stars: formation

\section{INTRODUCTION}

Bipolar molecular outflows have been identified as a common occurrence in the formation of low-mass stars (e.g., Bachiller \& Tafalla 1999; Richer et al. 2000). The main competing theoretical models proposed to explain these outflows invoke either narrowly collimated jets (e.g., Masson \& Chernin 1993; Raga \& Cabrit 1993) or wide-angle winds (Shu et al. 1991; Shu et al. 2000) to sweep up and sometimes shock ambient material. In either case, the jet or wind is thought to arise as a consequence of the accretion of matter onto the central star through a circumstellar disk. Matter in the disk diverted away from the star is collimated by magnetic

\footnotetext{
${ }^{1}$ Based at the Institute of Astronomy and Astrophysics, Academia Sinica, P.O. Box 23-141, Taipei 106, Taiwan.
}

fields to form the observed jet or wind. This jet or wind carries away excess angular momentum, thereby allowing material to accrete onto the central protostar, and may play an important role in halting accretion by clearing away infalling material.

Bipolar molecular outflows also are commonly found around young high-mass stars (Shepherd \& Churchwell 1996; Zhang et al. 2001). These outflows are generally much more massive and energetic than those from low-mass stars. Here, however, we lack a basic understanding of how these outflows are driven or their role in the star formation process. Accretion-based models for forming high-mass stars face the problem that, once the central protostar attains a mass of about $10 M_{\odot}$, radiation pressure on dust can retard and perhaps even reverse the infalling material (for a brief review, see Stahler, Palla, \& Ho 2000). This problem is further exacerbated if contraction of the central protostar contributes significantly to, or even dominates, 
TABLE 1

TARget Sources

\begin{tabular}{|c|c|c|c|c|c|c|}
\hline \multirow[b]{2}{*}{ IRAS NAME } & \multicolumn{2}{|c|}{$\begin{array}{l}\text { Position } \\
\text { (J2000.0) }\end{array}$} & \multirow{2}{*}{$\begin{array}{c}\text { Distance } \\
(\mathrm{kpc})\end{array}$} & \multirow{2}{*}{$\begin{array}{l}\text { Luminosity } \\
\quad\left(10^{4} L_{\odot}\right)\end{array}$} & \multirow{2}{*}{$\begin{array}{c}V_{\mathrm{lsr}} \\
\left(\mathrm{km} \mathrm{s}^{-1}\right)\end{array}$} & \multirow[b]{2}{*}{ OTHER NAME ${ }^{\mathrm{a}}$} \\
\hline & $\alpha$ & $\delta$ & & & & \\
\hline $21519+5613 \ldots \ldots \ldots$ & 215339.23 & +562745.5 & 7.3 & 1.9 & -63.2 & Mol 139 \\
\hline $22506+5944 \ldots \ldots \ldots$ & 225238.65 & +600055.7 & 5.7 & 2.2 & -51.5 & Mol 151 \\
\hline
\end{tabular}

Nоте.-Units of right ascension are hours, minutes, and seconds, and units of declination are degrees, arcminutes, and arcseconds.

${ }^{\text {a }}$ From on Molinari et al. 1996.

the total luminosity. To form a massive star on a timescale that is short compared with its Kelvin-Helmholtz contraction timescale, the mass-accretion rate onto massive protostars must be orders of magnitude higher than that for low-mass protostars. Both these difficulties have stimulated radically different models for forming high-mass stars, such as the coalescence of low-mass protostars (Bonnell, Bate, \& Zinnecker 1998). Models based on coalescing protostars would naturally predict uncollimated or even disrupted outflows, whereas those based on accretion might lead to more collimated outflows.

Recent surveys with single-dish telescopes show that massive outflows are commonly associated with UC-H II regions (Shepherd \& Churchwell 1996; Zhang et al. 2001), as well as their possible precursors (Zhang et al. 2001). In the vast majority of cases, however, it is not clear whether a given outflow is driven by a single star and, if so, the nature of this star. Nor has the spatial-kinematic structure of most massive outflows been studied in sufficient detail to place firm constraints on possible driving mechanisms. Because young massive stars usually form in close association with other stars and lie at comparatively large distances, high angular resolution observations with interferometers are necessary to overcome source confusion and reveal the detailed properties of the outflow and its driving source. Among known outflows associated with luminous young stellar objects (YSOs), only about 10 or so have been studied with interferometers. High angular resolution observations of more examples, in particular promising candidates for highmass protostars, are needed to better understand both the common features and individual variations seen in outflows from luminous YSOs.

Here we report observations of the bipolar molecular outflows associated with the two luminous far-IR sources, IRAS $21519+5613$ and IRAS $22506+5944$, as well as the dust and molecular-gas condensations on which these outflows appear to be centered. Both these sources have been proposed to be precursors of UC-H II regions by Molinari et al. $(1996,1998)$. IRAS $21519+5613$ and IRAS $22506+$ 5944 have comparable far-IR luminosities of $1.9 \times 10^{4}$ and $2.2 \times 10^{4} L_{\odot}$, respectively, at their inferred distances of 7.3 and $5.7 \mathrm{kpc}$. Yet, despite their high luminosities, no radio emission at $6 \mathrm{~cm}$ was detected toward either source at a $3 \sigma$ upper limit of $\sim 0.3 \mathrm{mJy}^{\text {beam }}{ }^{-1}$ in $\sim 3^{\prime \prime}$ beam (Molinari et al. 1998). This places an upper limit on the diameter of any UC-H II region (assuming that it is optically thick at $6 \mathrm{~cm}$ and has an electron temperature of $\sim 10,000 \mathrm{~K}$ ) of $\sim 0.001 \mathrm{pc}$, 2 orders of magnitude smaller than the typical size of $\mathrm{UC}-\mathrm{H}$ II regions. Both sources exhibit water maser activity and are associated with dense molecular gas traced by ammonia and CS $(J=2-1)$ (Wouterloot, Brand, \& Fiegle
1993; Molinari et al. 1996; Bronfman, Nyman, \& May 1996; Migenes et al. 1999), which together with the abovementioned properties make them credible candidates for highmass protostars.

The outflows from IRAS $21519+5613$ and IRAS $22506+$ 5944 show a clear bipolar morphology in $\mathrm{CO}(J=2-1)$ as observed at an angular resolution of $\sim 30^{\prime \prime}$ with the NRAO 12 $\mathrm{m}$ telescope (Zhang et al. 2001). Although these outflows have among the best-separated lobes in the sample observed by Zhang et al. (2001), little else can be discerned of their spatialkinematic structure. Here we report higher angular resolution interferometric observations in ${ }^{12} \mathrm{CO}(J=1-0),{ }^{13} \mathrm{CO}(J=$ $1-0), \mathrm{C}^{18} \mathrm{O}(J=1-0)$, and $3 \mathrm{~mm}$ continuum. To recover the extended emission near the central velocity of the ambient cloud filtered out by the interferometer, we also conducted single-dish observations in ${ }^{12} \mathrm{CO}(J=1-0)$ and ${ }^{13} \mathrm{CO}(J=$ $1-0)$. In $\S 2$ we describe the observations and data reduction. In $\S 3$ we present results of the dust and molecular gas condensation from the $3 \mathrm{~mm}$ continuum and $\mathrm{C}^{18} \mathrm{O}$ observations. In $\S 4$ we present images of the molecular outflows and derive their physical properties. In $\S 5$ we discuss the implications of our results for the nature of outflow driving sources and outflow-driving agents. We conclude with a summary of the main points in $\S 6$.

\section{OBSERVATIONS AND DATA REDUCTION}

\subsection{BIMA Array and NRAO $12 \mathrm{~m}$ Telescope}

Pertinent information on the target sources, including position, distance, luminosity, and systemic velocity $\left(V_{\mathrm{lsr}}\right)$, is summarized in Table 1. We conducted interferometric observations of these sources between 1999 May and July with the Berkeley-Illinois-Maryland Association (BIMA) array, which comprises $106.1 \mathrm{~m}$ dishes. The ${ }^{12} \mathrm{CO}(J=1-0)$ transition was observed in the most compact $\mathrm{D}$ configuration as well as in the more extended $\mathrm{C}$ configuration. The ${ }^{13} \mathrm{CO}(J=1-0)$ and $\mathrm{C}^{18} \mathrm{O}(J=1-0)$ transitions were observed simultaneously in the higher angular resolution $\mathrm{C}$ configuration only. These observations yielded images with angular resolutions of about $6^{\prime \prime}-10^{\prime \prime}$ over a field of diameter $2^{\prime}$ (i.e., the half-power beam width [HPBW] of the primary beam of the BIMA array). In ${ }^{12} \mathrm{CO}$ and ${ }^{13} \mathrm{CO}$, we configured the correlator to provide 128 channels over a bandwidth of $50 \mathrm{MHz}$, corresponding to a velocity resolution of $1.0 \mathrm{~km} \mathrm{~s}^{-1}$ spanning a velocity range of $\sim 130 \mathrm{~km} \mathrm{~s}^{-1}$. In $\mathrm{C}^{18} \mathrm{O}$, the correlator was configured to provide a higher velocity resolution of $0.27 \mathrm{~km}$ $\mathrm{s}^{-1}$ spanning a smaller velocity range of $68 \mathrm{~km} \mathrm{~s}^{-1}$. To measure the $3 \mathrm{~mm}$ continuum emission, we used data in the line-free parts of the spectrum from the ${ }^{12} \mathrm{CO}(J=1-0)$ observations, corresponding to a total bandwidth of $800 \mathrm{MHz}$ 

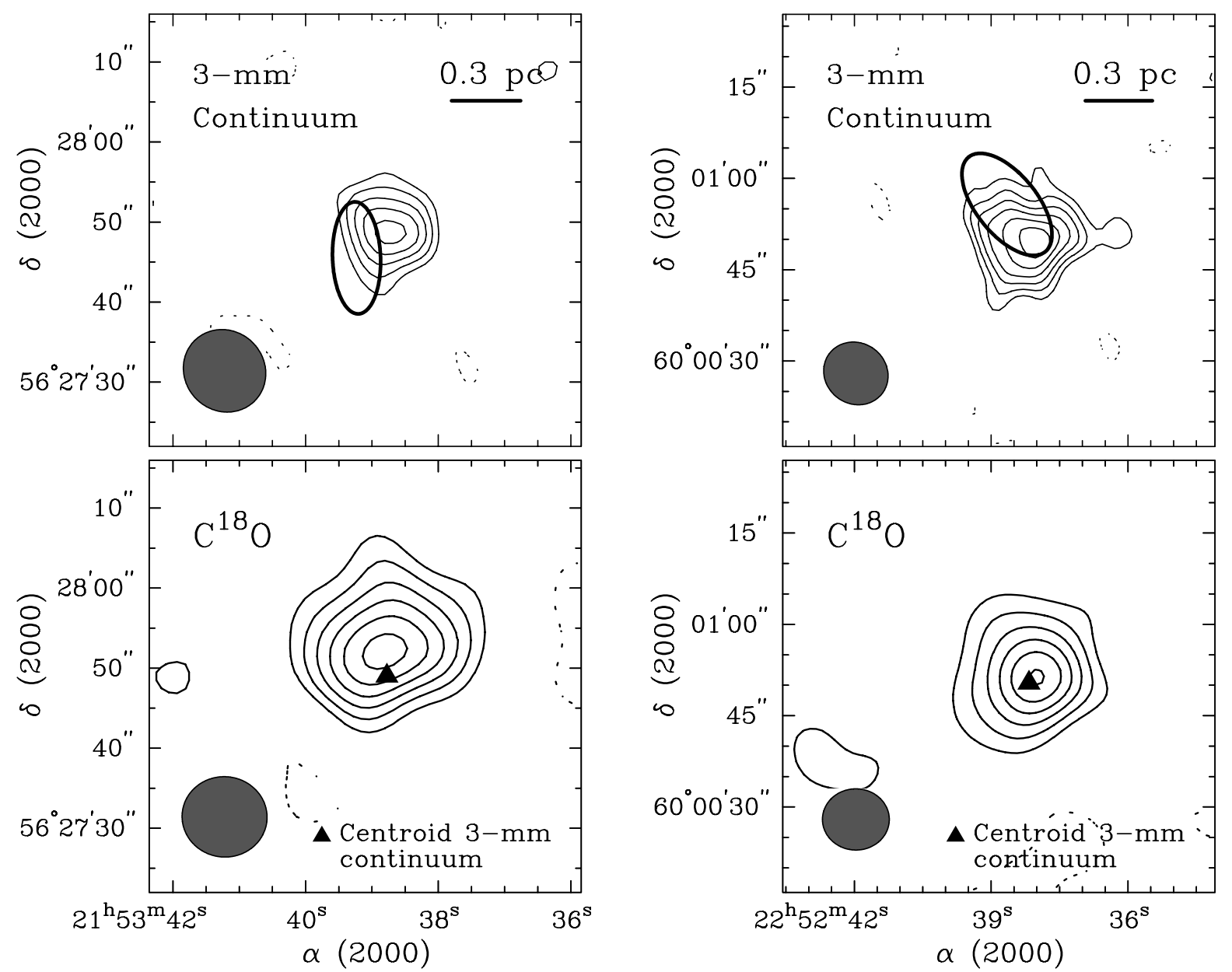

FIG. 1.-Top left: $3 \mathrm{~mm}$ continuum emission of IRAS 21519+5613, with contour levels at -3 (dotted line), 3, 4, 5, 6, and $7 \times 2.5 \mathrm{mJy}^{\text {beam }}{ }^{-1}$. The ellipse marks the $1 \sigma$ positional uncertainty of the IRAS source. Bottom left: $\mathrm{C}^{18} \mathrm{O}$ emission integrated from -66.8 to $-61.5 \mathrm{~km} \mathrm{~s}^{-1}$, with contour levels at -0.7 (dotted line) and from 0.7 to 2.45 in steps of $0.35 \mathrm{Jy}_{\text {beam }}^{-1} \mathrm{~km} \mathrm{~s}^{-1}$. The centroid of the $3 \mathrm{~mm}$ continuum is indicated by a triangle. The dark ellipse in each panel denotes the synthesized beam. Right: Same as above but for IRAS $22506+5944$. Contour levels are $-3,3,4,5,6,7$, and $8 \times 3 \mathrm{mJy}^{-1}$ beam ${ }^{-1}$ in the top panel and $-1.2,1.2$, to 4.2 in steps of $0.6 \mathrm{Jy} \mathrm{beam}^{-1} \mathrm{~km} \mathrm{~s}^{-1}$ in the bottom panel. The $\mathrm{C}^{18} \mathrm{O}$ emission is integrated from -54.0 to $-49.7 \mathrm{~km} \mathrm{~s}^{-1}$.

centered at $113.8 \mathrm{GHz}$. The absolute flux density scale was set from observations of a planet and is estimated to have an accuracy of $\sim 20 \%$. The quasar $2038+513$ was observed as the secondary calibrator for both target sources. We calibrated the data using the MIRIAD package.

In our images, we found that spatially extended emission at and near the systemic velocity of the ambient molecular cloud was filtered out by the BIMA array. This problem is especially severe in ${ }^{12} \mathrm{CO}$ and ${ }^{13} \mathrm{CO}$, and if ignored it will compromise measurements of the outflow mass at low velocities. We therefore obtained single-dish data in ${ }^{12} \mathrm{CO}$ and ${ }^{13} \mathrm{CO}$ with the National Radio Astronomy Observatory ${ }^{2}$ (NRAO) $12 \mathrm{~m}$ telescope on 2000 June 14-17 to recover the missing short-spacing information. In both these lines, we mapped a field $2.9 \times 2 . ' 9$ in size divided into a $7 \times 7$ grid with grid points separated by $25^{\prime \prime}$. This provided full Nyquist sampling over a field with dimensions 1.5 times the HPBW of the BIMA array. These observations were performed in position-switching mode for ${ }^{12} \mathrm{CO}$ $(J=1-0)$ and frequency-switching mode for ${ }^{13} \mathrm{CO}(J=$ $1-0)$. We reduced the data using the CLASS package.

\footnotetext{
${ }^{2}$ The National Radio Astronomy Observatory is a facility of the National Science Foundation operated under cooperative agreement by Associated Universities, Inc.
}

\subsection{Combining Single-Dish and Interferometric Data}

The single-dish and interferometer data were combined using the procedure described by Vogel et al. (1984), whereby visibility data are first generated from the single-dish data and then combined with the interferometer visibilities. Below we outline the key steps, all performed using the MIRIAD package. First, the primary beam of the NRAO $12 \mathrm{~m}$ telescope of $\sim 55^{\prime \prime}$ was deconvolved from the single-dish map using the CLEAN algorithm. Second, the deconvolved single-dish map was convolved with the primary beam of the BIMA antenna, which as mentioned earlier has a HPBW of $\sim 2^{\prime}$. Third, we applied a Fourier transform to the single-dish map to convert the data into equivalent interferometric visibilities. Finally, the visibilities from both the NRAO $12 \mathrm{~m}$ telescope and BIMAarray were combined in the mapping procedure to make the images shown in Figures 2-7.

\section{DUST AND MOLECULAR GAS CONDENSATION}

\subsection{Structure and Kinematics}

Figure 1 shows contour maps of the $3 \mathrm{~mm}$ continuum and integrated $\mathrm{C}^{18} \mathrm{O}$ emission detected toward IRAS $21519+5613$ and IRAS $22506+5944$. The $3 \mathrm{~mm}$ continuum maps incorporate data from both the $\mathrm{C}$ and $\mathrm{D}$ configurations of the BIMA 
TABLE 2

The Core Properties

\begin{tabular}{|c|c|c|c|c|c|}
\hline \multirow[b]{2}{*}{ IRAS NAME } & \multirow[b]{2}{*}{ EMISSION } & \multicolumn{2}{|c|}{$\begin{array}{l}\text { Position } \\
\text { (J2000.0) }\end{array}$} & \multirow[b]{2}{*}{ Integrated Flux Density } & \multirow{2}{*}{$\begin{array}{l}\text { MASS } \\
\left(M_{\odot}\right)\end{array}$} \\
\hline & & $\alpha$ & $\delta$ & & \\
\hline \multirow[t]{2}{*}{$21519+5613 \ldots \ldots$} & $3 \mathrm{~mm}$ continuum & 215338.77 & +562749.0 & $17.7 \mathrm{mJy}$ & 200 \\
\hline & $\mathrm{C}^{18} \mathrm{O}$ & 215338.80 & +562752.4 & $5.4 \mathrm{Jy} \mathrm{km} \mathrm{s}^{-1}$ & 190 \\
\hline \multirow[t]{2}{*}{$22506+5944 \ldots \ldots$} & $3 \mathrm{~mm}$ continuum & 225238.17 & +600050.3 & $48.4 \mathrm{mJy}$ & 260 \\
\hline & $\mathrm{C}^{18} \mathrm{O}$ & 225238.02 & +600051.4 & $9.9 \mathrm{Jy} \mathrm{km} \mathrm{s}^{-1}$ & 260 \\
\hline
\end{tabular}

NoTE.-Units of right ascension are hours, minutes, and seconds, and units of declination are degrees, arcminutes, and arcseconds.

array. For the $\mathrm{C}^{18} \mathrm{O}$ maps, where we have data in the higher angular resolution C configuration only, we applied a Gaussian taper to the visibilities to obtain a synthesized beam of $\sim 10^{\prime \prime}$, similar to that in the $3 \mathrm{~mm}$ continuum. The $\mathrm{C}^{18} \mathrm{O}$ line width of both sources is $\sim 4 \mathrm{~km} \mathrm{~s}^{-1}$ at FWHM, and the emission has a spatial distribution similar to that seen in ${ }^{13} \mathrm{CO}$ at the same velocities (Figs. 2 and 5). The $3 \mathrm{~mm}$ continuum and $\mathrm{C}^{18} \mathrm{O}$ emission of both sources are at best only marginally resolved and have centroids that agree to within a few arcseconds, smaller than the size of the synthesized beam. Their positions and integrated intensities are listed in Table 2.

Both the $3 \mathrm{~mm}$ continuum and $\mathrm{C}^{18} \mathrm{O}$ emission likely trace a condensation of dust and gas at the center of each outflow (see $\S 4)$. Although the $3 \mathrm{~mm}$ continuum could also be produced by free-free emission from ionized gas, here the required emission measure of $\gtrsim 10^{10} \mathrm{pc} \mathrm{cm}^{-6}$ is at least 3 orders of magnitude larger than is usually the case for $\mathrm{UC}-\mathrm{H}$ II regions. Furthermore, as shown below, the mass of the condensation derived from the $3 \mathrm{~mm}$ continuum is comparable with that derived from the $\mathrm{C}^{18} \mathrm{O}$ line assuming that the former is produced entirely (or predominantly) by dust.

Recently, Molinari et al. (2002) observed IRAS 21519+ 5613 with the Owen Valley Radio Observatory (OVRO). They reported a total $3 \mathrm{~mm}$ continuum flux of $\sim 21 \mathrm{mJy}$, in close agreement with our measured value of $\sim 18 \mathrm{mJy}$. Their observations at higher angular resolution $\left(\sim 4^{\prime \prime}\right)$ revealed two components, with the minor component lying $\sim 4^{\prime \prime}$ north of the main component and having a flux density of $\sim 6 \mathrm{mJy}$. We associate the main $3 \mathrm{~mm}$ component with the centroid of our observed $3 \mathrm{~mm}$ continuum source. Molinari et al. (2002) also observed IRAS $21519+5613$ in the $1 \mathrm{~mm}$ continuum and $\mathrm{HCO}^{+}$and found that both are coincident with the main $3 \mathrm{~mm}$ component. The spectral index of $\sim 2.5$ between 3 and $1 \mathrm{~mm}$ is consistent with emission from dust.

\subsection{Mass}

From the $3 \mathrm{~mm}$ continuum, an approximate mass for the gas and dust condensation (assuming a gas-to-dust ratio of 100) can be derived from the relationship $M_{\text {gas }+ \text { dust }}=\left(F_{\nu} D^{2}\right)$ / $\left[B_{\nu}\left(T_{d}\right) \kappa_{\nu}\right]$ (Hildebrand 1983), where $F_{\nu}$ is the flux density of the dust emission at frequency $\nu, D$ is the distance to the source, and $B_{\nu}$ is the Planck function at a dust temperature $T_{d}$. The dust opacity per gram of gas is taken to be $\kappa_{\nu}=$ $0.006(\nu / 245 \mathrm{GHz}){ }^{\beta} \mathrm{cm}^{2} \mathrm{~g}^{-1}$ (Kramer et al. 1998; Shepherd $\&$ Watson 2002), where $\beta$ is the opacity index. We assume $\beta=1.5$ for both sources, and a (uniform) dust temperature equal to the measured gas temperature. The latter is derived from the peak brightness temperature of the optically thick $\mathrm{CO}$ emission at the ambient cloud velocity, corresponding to $\sim 30 \mathrm{~K}$ for IRAS $21519+5613$ and $\sim 40 \mathrm{~K}$ for IRAS $22506+5944$. We find a total mass for the dust and gas condensation of $\sim 200 M_{\odot}$ for IRAS $21519+5613$ and $\sim 260 M_{\odot}$ for IRAS $22506+5944$. The largest uncertainty in this estimate probably arises from the adopted dust opacity. If $\beta=2$, the estimated mass becomes 290 and $380 M_{\odot}$ for IRAS $21519+5613$ and IRAS $22506+5944$ respectively, about 1.5 times higher than in the case of $\beta=1.5$. The assumed dust temperature also affects the estimated mass, with the product of temperature and mass being constant.

To calculate the mass of molecular gas associated with the $\mathrm{C}^{18} \mathrm{O}$ emission, we follow the method described by Scoville et al. (1986). Because the ${ }^{13} \mathrm{CO}$ emission is optically thin (see $\S 4.2$ ), we assume the $\mathrm{C}^{18} \mathrm{O}$ emission also to be optically thin and in LTE, as well as an abundance ratio $\left[\mathrm{H}_{2}\right] /\left[{ }^{12} \mathrm{CO}\right]=10^{4}$. Given a distance to galactic center, $D_{\mathrm{GC}}$, of $\sim 11.5 \mathrm{kpc}$ for both sources, we adopt an abundance ratio $\left.{ }^{12} \mathrm{CO}\right] /\left[\mathrm{C}^{18} \mathrm{O}\right]=$ 713 as estimated from the relationship $\left[{ }^{12} \mathrm{CO}\right] /\left[\mathrm{C}^{18} \mathrm{O}\right]=$ $(58.8 \pm 11.8) D_{\mathrm{GC}}+(37.1 \pm 82.6)$ (Wilson \& Rood 1994), where $D_{\mathrm{GC}}$ is in kpc. Assuming the gas temperature as described above, we derive a total mass for the molecular gas of $190 M_{\odot}$ for IRAS $21519+5613$ and $260 M_{\odot}$ for IRAS 22506+5944. The condensation masses derived from $\mathrm{C}^{18} \mathrm{O}$ are therefore in close agreement with those derived from the $3 \mathrm{~mm}$ continuum, supporting our earlier argument that the $3 \mathrm{~mm}$ continuum is likely produced by dust. The derived dust and molecular gas masses are summarized in Table 2. The deconvolved size of the dust and gas condensations is $\lesssim 0.4$ pc for both IRAS $21519+5613$ and IRAS $22506+5944$. The masses and sizes of the condensations are therefore comparable with those associated with other luminous YSOs (e.g., Cesaroni et al. 1997; Shepherd \& Kurtz 1999; Molinari et al. 2002).

\section{BIPOLAR MOLECULAR OUTFLOWS}

\subsection{Structure and Kinematics}

\subsubsection{IRAS 21519+5613}

Figures 2 and 3 show channel maps of the ${ }^{13} \mathrm{CO}$ and ${ }^{12} \mathrm{CO}$ emission, respectively, associated with IRAS $21519+5613$. For display purposes, we have smoothed the ${ }^{12} \mathrm{CO}$ channel maps to a velocity resolution of $2.04 \mathrm{~km} \mathrm{~s}^{-1}$. The ${ }^{12} \mathrm{CO}$ emission near the systemic velocity $\left(V_{\mathrm{lsr}} \pm 2 \mathrm{~km} \mathrm{~s}^{-1}\right)$ fills the entire primary beam. Away from these velocities, both the ${ }^{12} \mathrm{CO}$ and ${ }^{13} \mathrm{CO}$ emissions are extended in the east-west direction and show a velocity pattern characteristic of a bipolar molecular outflow. The origin of the outflow appears to be closely coincident with the centroid of the molecular gas and dust condensation described in $\S 3$. In ${ }^{12} \mathrm{CO}$, this outflow can 


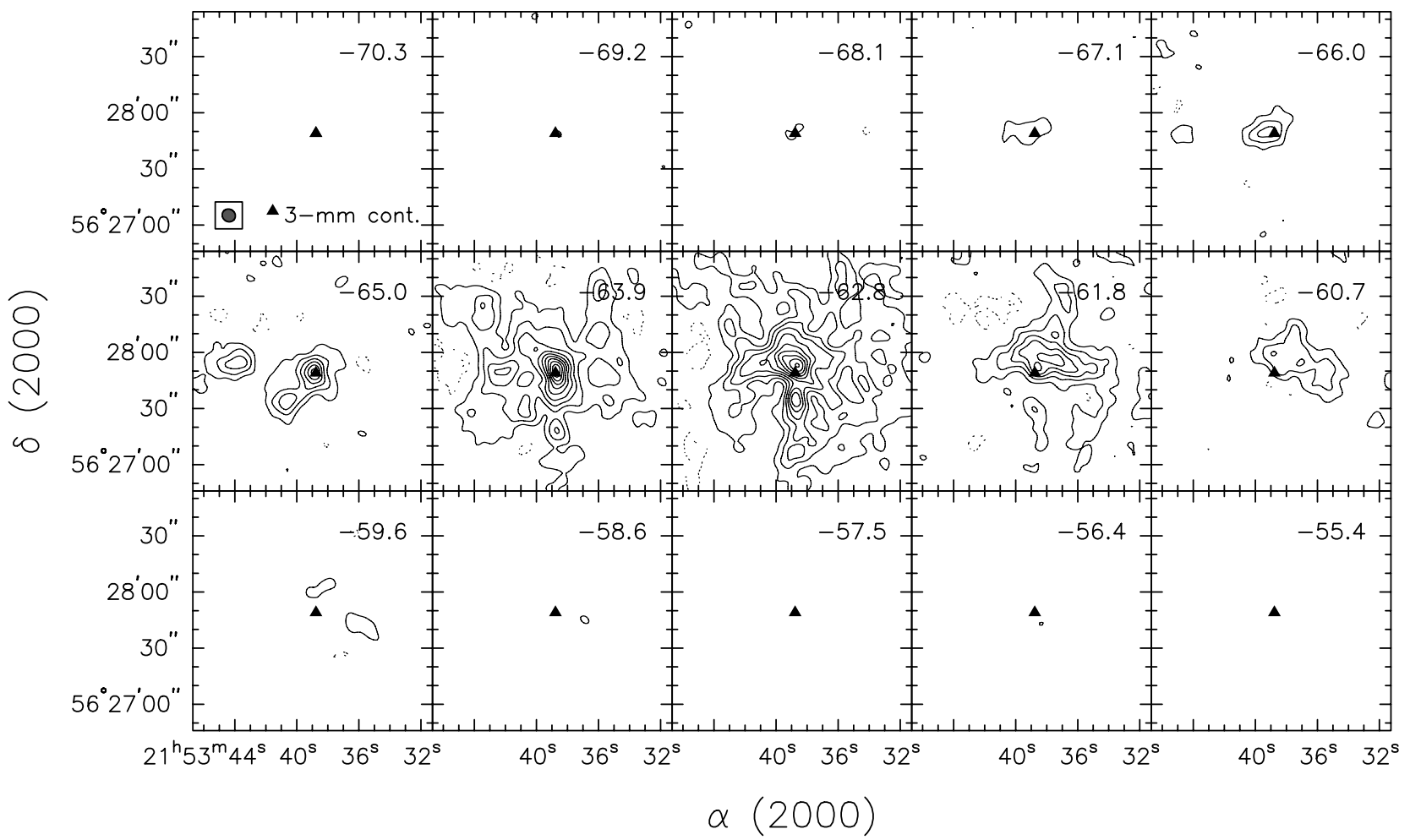

FIG. 2. Channel maps of IRAS $21519+5613$ in ${ }^{13} \mathrm{CO}$. The local standard of rest velocity of each channel is indicated in the upper right-hand corner of each panel. The rms noise $(\sigma)$ is $0.08 \mathrm{Jy}_{\text {beam }}{ }^{-1}$. Solid contours are from 0.32 to 4.64 in steps of $0.48 \mathrm{Jy} \mathrm{beam}^{-1}$, and dotted contours indicate -0.32 and $-0.80 \mathrm{Jy}$ beam $^{-1}$. The synthesized beam is shown in the lower left-hand corner of the first panel, and the triangle marks the centroid of the $3 \mathrm{~mm}$ continuum.

be traced to velocities $v_{\text {flow }} \approx 16 \mathrm{~km} \mathrm{~s}^{-1}$ from the systemic velocity, where $v_{\text {flow }} \equiv\left|V_{\text {flow }}-V_{\text {lsr }}\right|$ with $V_{\text {flow }}$ the measured flow velocity and $V_{\mathrm{lsr}}$ the systemic velocity of the source. (We use the notation $V$ for the measured velocity and $v$ for the velocity relative to the systemic velocity.) In the weaker
${ }^{13} \mathrm{CO}$ emission, the outflow can be detected only to velocities $v_{\text {flow }} \approx 5 \mathrm{~km} \mathrm{~s}^{-1}$.

The integrated blue- and redshifted ${ }^{12} \mathrm{CO}(J=1-0)$ emissions are shown in Figure 4, where the top panel corresponds to emission integrated over the entire line wing

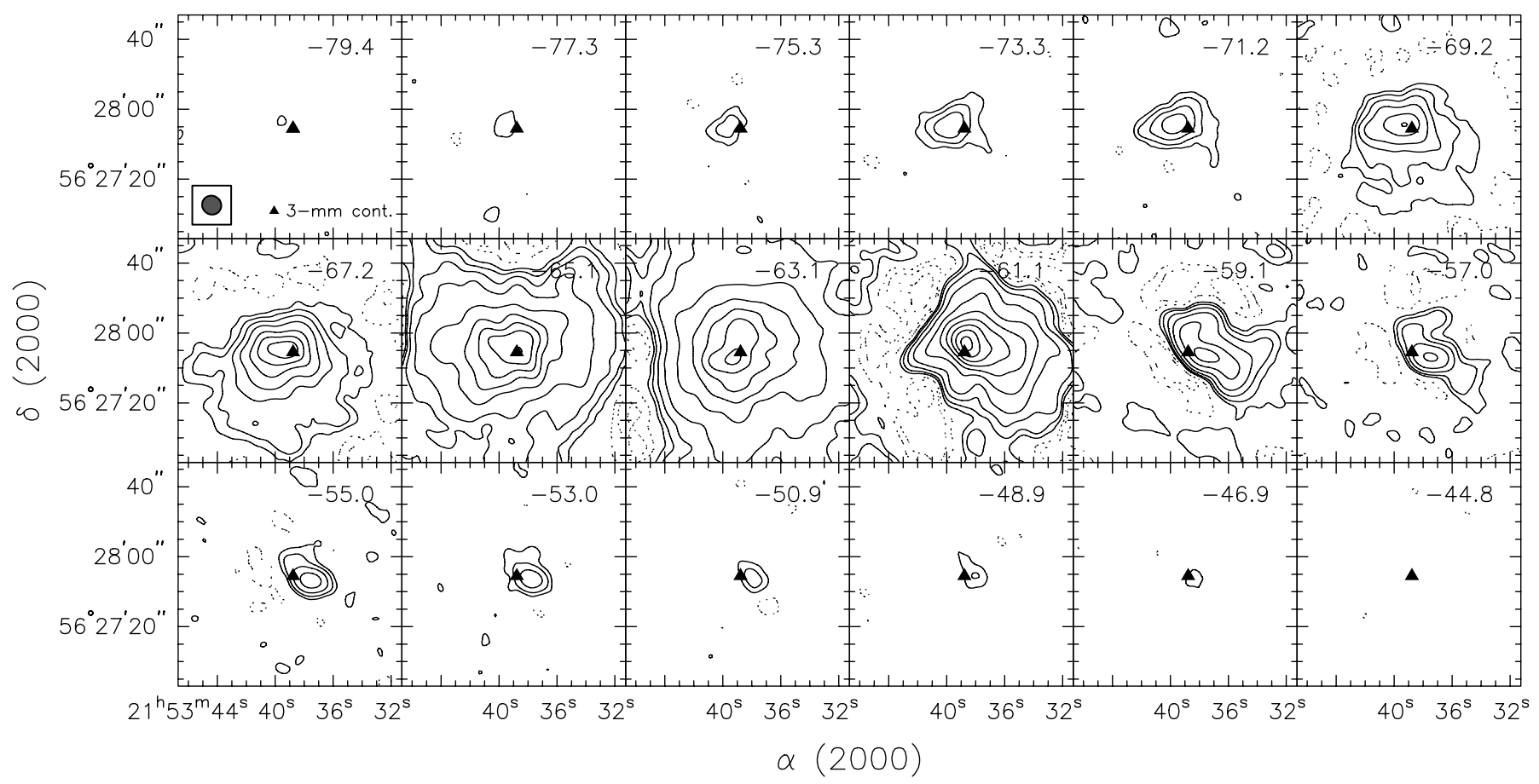

Fig. 3. - Same as in Fig. 2, but for ${ }^{12} \mathrm{CO}$. Solid contours are at 3, 7, 15, 30, 60, 90, 120, 150, 180, and $210 \times \sigma$, and dotted contours indicate -3 , -7 , and $-15 \times \sigma$, where $\sigma=0.12 \mathrm{Jy}^{\text {beam }}{ }^{-1}$. 


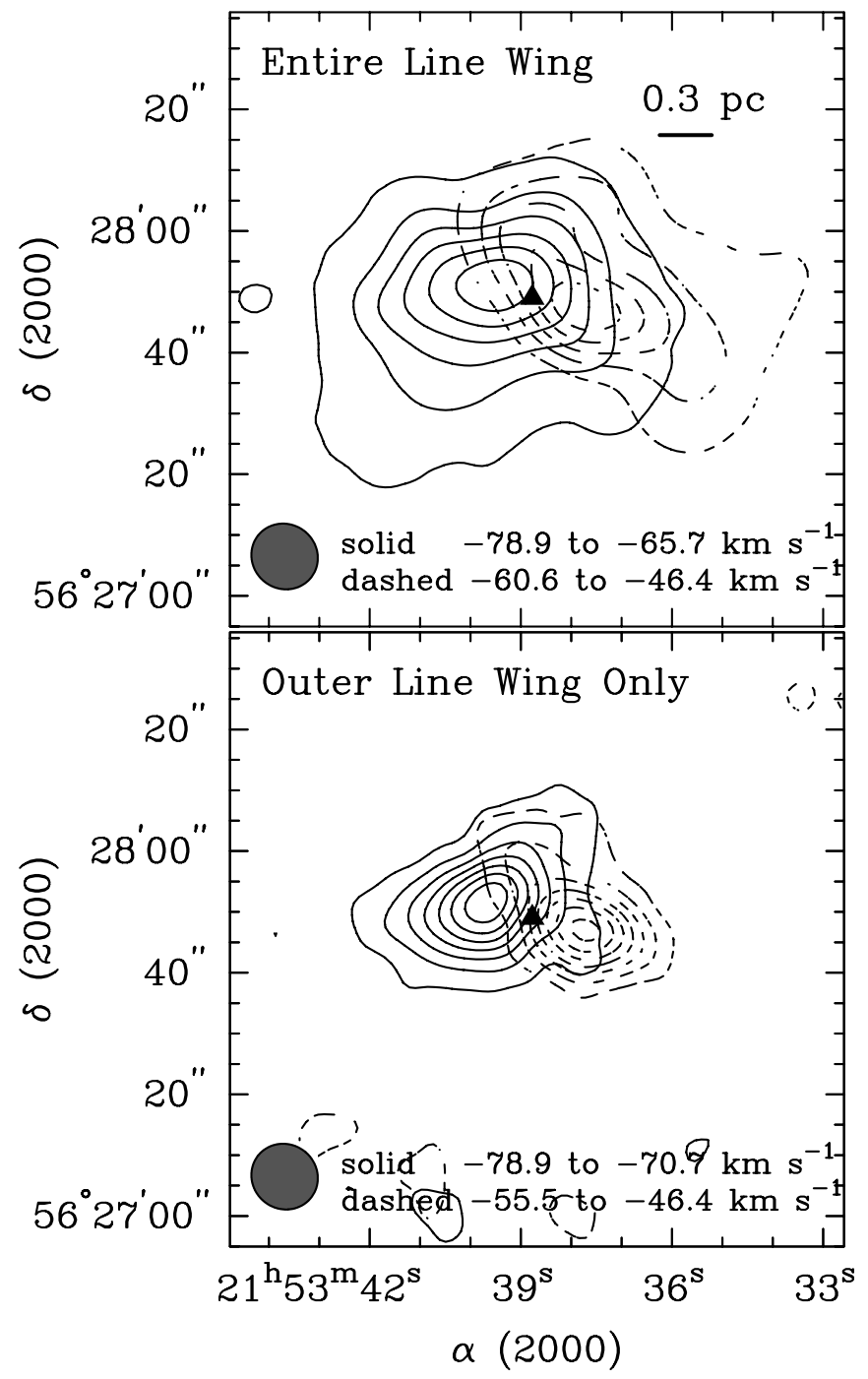

FIG. 4.-Bipolar molecular outflow of IRAS $21519+5613$ in ${ }^{12} \mathrm{CO}$ integrated over the entire line wing (top) and the outer line wing only (bottom). Blue- and redshifted emissions are shown by solid and dashed contours, respectively. Contours are from 10 to 85 in steps of $15 \mathrm{Jy}_{\text {beam }}^{-1} \mathrm{~km} \mathrm{~s}^{-1}$ in the top panel and from 1.5 to 19.5 in steps of $3 \mathrm{Jy} \mathrm{beam}^{-1} \mathrm{~km} \mathrm{~s}^{-1}$ in the bottom panel. The centroid of the $3 \mathrm{~mm}$ continuum is indicated by a triangle.

$\left(2.5 \lesssim v_{\text {flow }} \lesssim 16.0 \mathrm{~km} \mathrm{~s}^{-1}\right)$ and the bottom panel over the "outer" line wing $\left(7.6 \lesssim v_{\text {flow }} \lesssim 16.0 \mathrm{~km} \mathrm{~s}^{-1}\right)$ only. This figure illustrates the difficulty in fully separating the outflow at low velocities from the ambient gas. As can be seen in the top panel, the integrated ${ }^{12} \mathrm{CO}$ emission over the entire line wing exhibits an extended structure to the south in both bipolar lobes. No such extension, however, is seen in the bottom panel, where only emission at higher velocities is included. A close examination of the ${ }^{12} \mathrm{CO}$ channel maps in Figure 3 reinforces the notion that this extended morphology is probably not associated with the outflow but instead is likely caused by low-velocity ambient cloud emission.

The position angle defined by the centroids of the blue- and redshifted lobes of the outflow is $\sim 75^{\circ}$. The centroid of the $3 \mathrm{~mm}$ continuum dust and $\mathrm{C}^{18} \mathrm{O}$ molecular gas condensation lies between the centroids of the outflow lobes, suggesting that it marks the location of the driving source. On the other hand, as measured along their individual major axes, the western (redshifted) lobe has a position angle of $\sim 55^{\circ}$ whereas the eastern (blueshifted) lobe has a position angle of $\sim 120^{\circ}$; furthermore, these axes do not intersect at the centroid of the dust and gas condensation. Note that these position angles are measured from the integrated ${ }^{12} \mathrm{CO}$ emission in the outer line wings only (Fig. 4, bottom panel), where the influence of ambient gas emission is less severe. The apparently different position angles of the major axes of the outflow lobes may result from the complex density structure of the molecular cloud cores into which the ${ }^{12} \mathrm{CO}$ outflow is being driven or swept up.

\subsubsection{IRAS $22506+5944$}

Figures 5 and 6 show channel maps of the ${ }^{13} \mathrm{CO}$ and ${ }^{12} \mathrm{CO}$ emission, respectively, associated with IRAS $22506+5944$. Again the ${ }^{12} \mathrm{CO}$ emission, but not this time the ${ }^{13} \mathrm{CO}$ emission, shows an east-west extension and a velocity pattern characteristic of a bipolar molecular outflow. The outflow in ${ }^{12} \mathrm{CO}$ is detectable to velocities $v_{\text {flow }} \approx 37 \mathrm{~km} \mathrm{~s}^{-1}$ in the redshifted and $v_{\text {flow }} \approx 56 \mathrm{~km} \mathrm{~s}^{-1}$ in the blueshifted lobes, more than 3 times higher than in the case of IRAS $21519+5613$. Because of the broad line profile, for display purposes we have smoothed the channel maps in Figure 6 to a velocity resolution of $4.07 \mathrm{~km} \mathrm{~s}^{-1}$. By comparison, the ${ }^{13} \mathrm{CO}$ emission extends to only $\pm 5 \mathrm{~km} \mathrm{~s}^{-1}$ of the systemic velocity and, unlike that of IRAS $21519+5613$, traces only the dust and gas condensation besides the surrounding ambient gas.

Figure 7 shows the integrated blue- and redshifted ${ }^{12} \mathrm{CO}$ $(J=1-0)$ emission, where the top panel corresponds to emission integrated over the entire line wing $\left(4.0 \lesssim v_{\text {flow }} \lesssim\right.$ $55.8 / 36.6 \mathrm{~km} \mathrm{~s}^{-1}$ for the blueshifted/redshifted lobes) and the bottom panel to the outer line wing $\left(9.1 \lesssim v_{\text {flow }} \lesssim\right.$ $55.8 / 36.6 \mathrm{~km} \mathrm{~s}^{-1}$ ) only. As before, the morphology of the outflow integrated over the entire line wing is contaminated by ambient gas emission. The centroid of the $3 \mathrm{~mm}$ dust continuum and $\mathrm{C}^{18} \mathrm{O}$ molecular gas condensation lies between the centroids of the blue- and redshifted outflow lobes. The major axes of the two lobes have different position angles, with the western (blueshifted) lobe having a position angle of $\sim 105^{\circ}$ and the eastern (redshifted) lobe a position angle of $\sim 70^{\circ}$. In this case, however, the major axes of the two lobes appear to intersect at the centroid of the dust and molecular gas condensation.

\subsection{Physical Properties}

For simplicity, when deriving the outflow mass from ${ }^{12} \mathrm{CO}$ measurements, the emission is usually assumed to be optically thin (e.g., Davis et al. 1998). This, however, can result in a severe underestimate of the outflow mass at velocities close to the systemic velocity, where the ${ }^{12} \mathrm{CO}$ is likely to be optically thick. Here we use the measured flux density in ${ }^{12} \mathrm{CO}$ and ${ }^{13} \mathrm{CO}$ to estimate the optical depth of the ${ }^{12} \mathrm{CO}$ emission at each velocity spatially averaged over the visible extent of the outflow (specifically, in a $25^{\prime \prime}$ beam centered on the respective sources). Ideally, one would also like to estimate the ${ }^{12} \mathrm{CO}$ opacity as a function of position, but this proved difficult given the limited angular resolution and sensitivity attained here. We expect the latter correction to be much less severe.

First, we note that the ${ }^{13} \mathrm{CO}$ emission is optically thin at all (detectable) velocities based on a comparison of the ratio in flux density of ${ }^{13} \mathrm{CO}$ and $\mathrm{C}^{18} \mathrm{O}$. The optical depth of the ${ }^{12} \mathrm{CO}$ emission at a given velocity can therefore be calculated from the equation

$$
\frac{\mathrm{S}_{\nu}\left({ }^{12} \mathrm{CO}\right)}{\mathrm{S}_{\nu}\left({ }^{13} \mathrm{CO}\right)}=\frac{\left[{ }^{12} \mathrm{CO}\right]}{\left[{ }^{13} \mathrm{CO}\right]}\left(\frac{1-e^{-\tau}}{\tau}\right),
$$




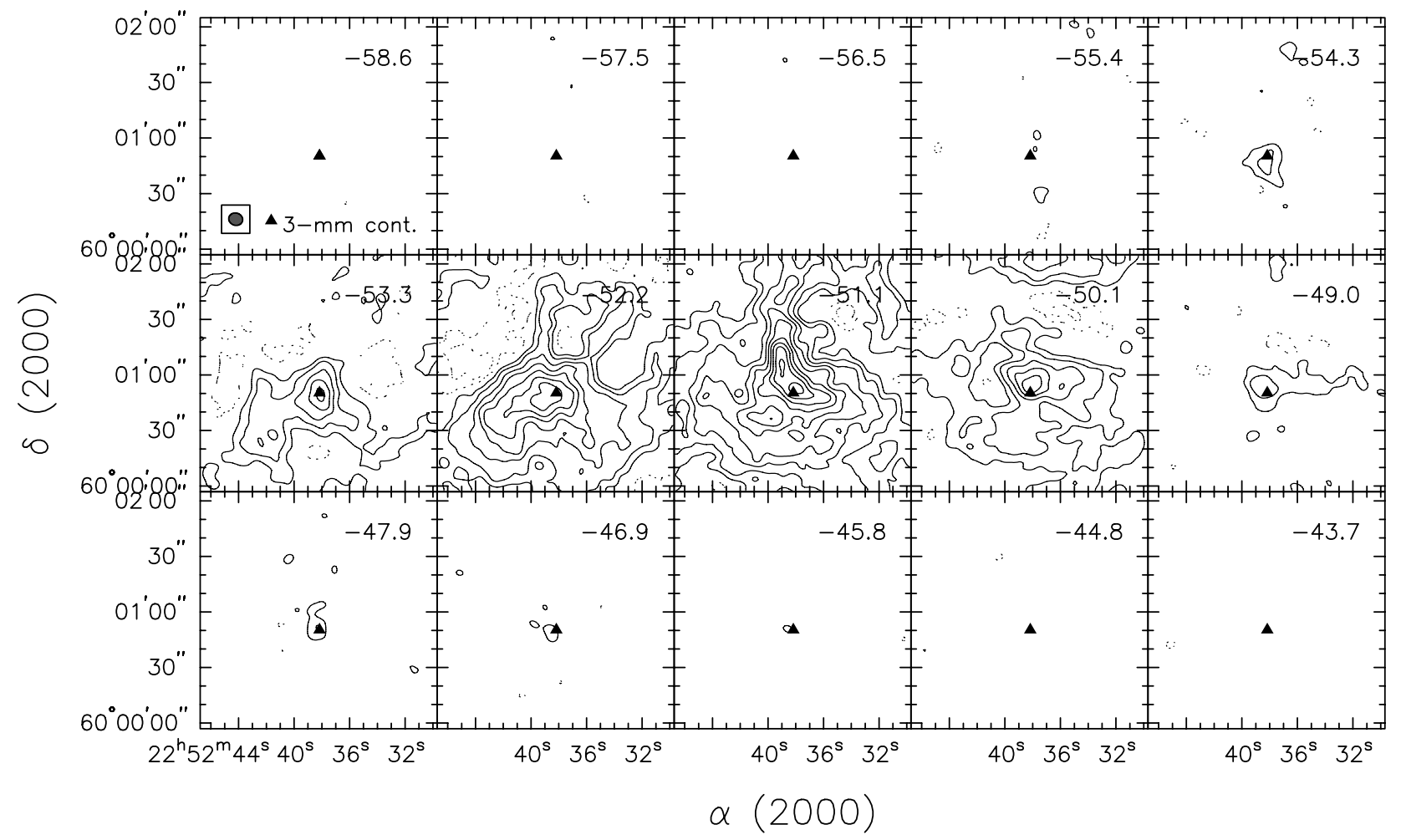

FIG. 5. - Same as in Fig. 2, but for IRAS $22506+5944$ in ${ }^{13} \mathrm{CO}$. Solid contours are at 4, 10, 20, 30, 40, 50, 60, 70, and $80 \times \sigma$, and dotted contours indicate -4 and $-10 \times \sigma$, where $\sigma=0.08 \mathrm{Jy}^{\text {beam }}{ }^{-1}$.

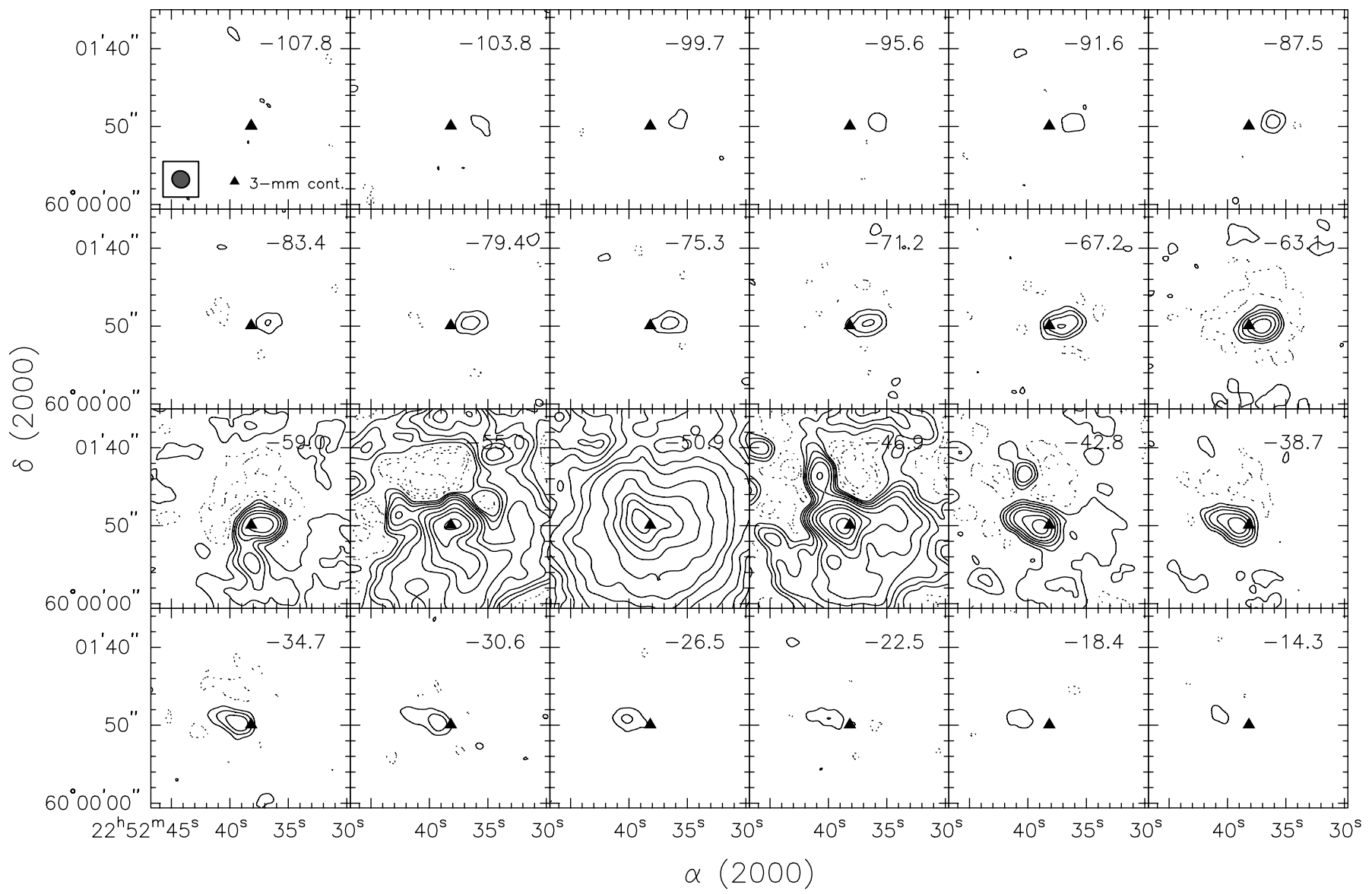

Fig. 6.-Same as in Fig. 2, but for IRAS $22506+5944$ in ${ }^{12}$ CO. Solid contours are at 3, 7, 12, 20, 30, 50, 75, 100, 150, 200, 250, 300, and $350 \times \sigma$, and dotted contours indicate $-3,-7,-12$, and $-20 \times \sigma$, where $\sigma=0.09 \mathrm{Jy} \mathrm{beam}^{-1}$. 


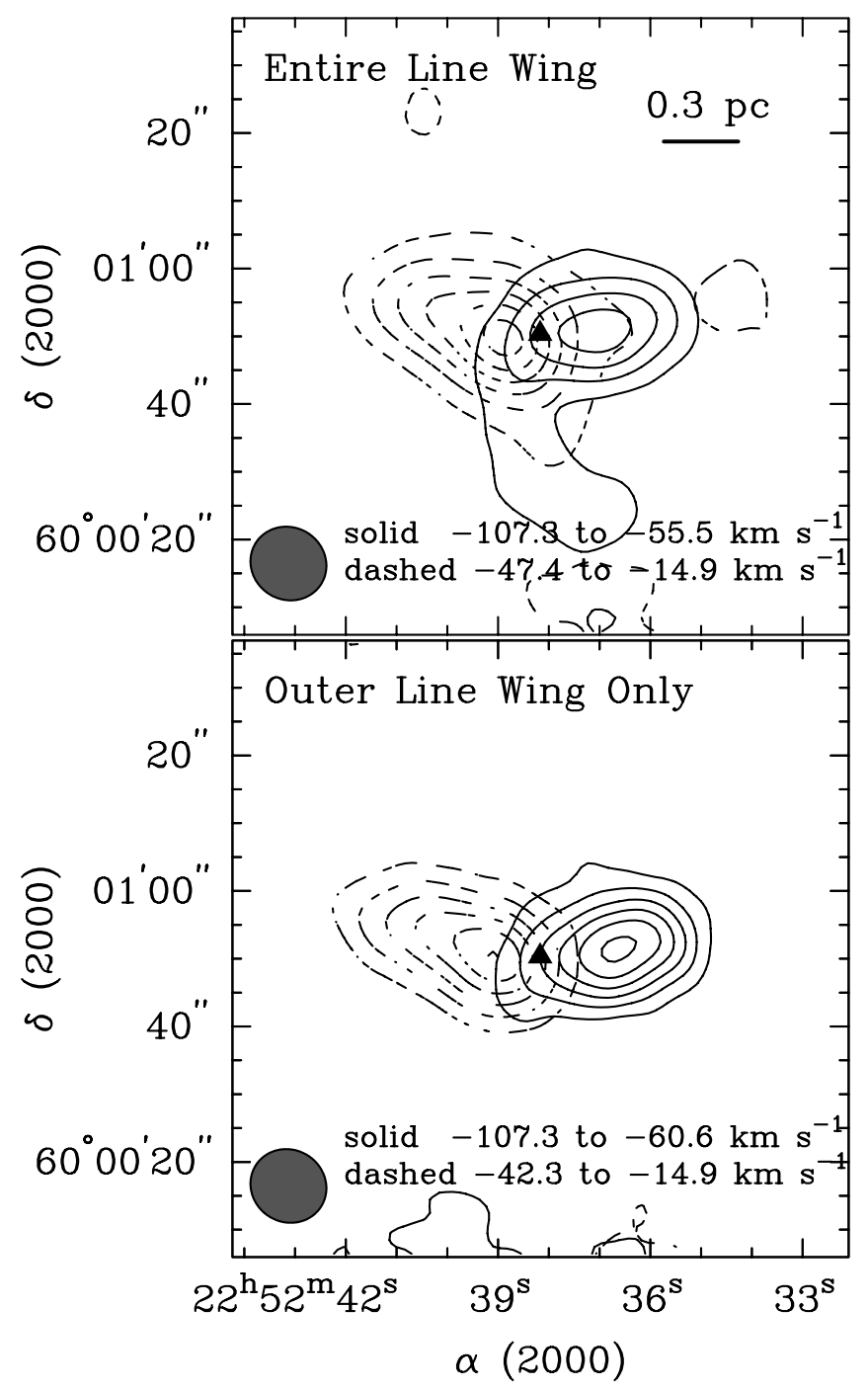

FIG. 7.- Same as in Fig. 4, but for IRAS $22506+5944$. Contours of ${ }^{12} \mathrm{CO}$ emission are from 12 to 112 in steps of $20 \mathrm{Jy} \mathrm{beam}^{-1} \mathrm{~km} \mathrm{~s}^{-1}$ in the top panel and from 4 to 44 in steps of $8 \mathrm{Jy}_{\text {beam }}^{-1} \mathrm{~km} \mathrm{~s}^{-1}$ in the bottom panel.

where $\left[{ }^{12} \mathrm{CO}\right]$ and $\left[{ }^{13} \mathrm{CO}\right]$ denote the abundances of the respective molecules. The abundance ratio $\left[{ }^{12} \mathrm{CO}\right] /\left[{ }^{13} \mathrm{CO}\right]$ can be estimated from the relationship $\left[{ }^{12} \mathrm{CO}\right] /\left[{ }^{13} \mathrm{CO}\right]=$ $(7.5 \pm 1.9) D_{\mathrm{GC}}+(7.6 \pm 12.9)$ found by Wilson \& Rood (1994), where $D_{\mathrm{GC}}$ is the galactocentric distance of the source in kpc. With $D_{\mathrm{GC}} \approx 11.5 \mathrm{kpc}$ for both sources, this gives $\left[{ }^{12} \mathrm{CO}\right] /\left[{ }^{13} \mathrm{CO}\right] \simeq 94$.

Figure 8 shows the derived opacity of ${ }^{12} \mathrm{CO}$ as a function of velocity for both IRAS $21519+5613$ and IRAS $22506+5944$. The opacity is evaluated over the velocity range $\sim V_{\text {lsr }} \pm 5 \mathrm{~km}$ $\mathrm{s}^{-1}$ only, corresponding to that over which ${ }^{13} \mathrm{CO}$ is detectable. We find that, for both sources, the ${ }^{12} \mathrm{CO}$ emission is very optically thick $(\tau \sim 10-30)$ within the velocity range $V_{\text {lsr }} \pm$ $2 \mathrm{~km} \mathrm{~s}^{-1}$ and still remains quite optically thick $(\tau \sim 1-2)$ up to the detectable velocity extent of the ${ }^{13} \mathrm{CO}$ emission. We calculate the outflow mass, $M_{\text {outflow }}$, from the equation

$$
M_{\text {outflow }}=\sum m\left(v_{i}\right)=\sum m^{\prime}\left(v_{i}\right)\left(\frac{\tau\left(v_{i}\right)}{1-e^{-\tau\left(v_{i}\right)}}\right),
$$

where $m^{\prime}\left(v_{i}\right)$ is the outflow mass at velocity $v_{i}$ assuming optically thin emission, $\tau\left(v_{i}\right)$ the optical depth at velocity $v_{i}$, and $m\left(v_{i}\right)$ the outflow mass after opacity correction. Beyond the velocity range over which the ${ }^{12} \mathrm{CO}$ opacity can be estimated, we assume the ${ }^{12} \mathrm{CO}$ emission to be optically thin (i.e., $\left.m\left(v_{i}\right)=m^{\prime}\left(v_{i}\right)\right)$. We further assume the gas to be in LTE and, as before, a (uniform) gas temperature of $\sim 30$ and $\sim 40 \mathrm{~K}$, respectively, for IRAS $21519+5613$ and IRAS $22506+5944$.

\subsubsection{IRAS $21519+5613$}

We derive a total molecular gas mass (i.e., both ambient gas and outflow) of $\sim 200 M_{\odot}$ for IRAS $21519+5613$ at velocities $\gtrsim 2.5 \mathrm{~km} \mathrm{~s}^{-1}$ from the systemic velocity, with a mass of $84 M_{\odot}$ at redshifted and $120 M_{\odot}$ at blueshifted velocities. At velocities closer to the systemic velocity, it is virtually impossible to distinguish the outflow from the ambient gas in the channel maps. Even at higher velocities, the surrounding ambient gas may still contribute significantly to the total gas mass. To better separate the ambient gas from the outflow at velocities where the former was deemed to contribute significantly to the total emission, we selected four regions adjacent to the outflow and computed their average flux density for each velocity channel, and then subtracted the derived value from that corresponding channel. This resulted in a corrected outflow mass of 62 and $80 M_{\odot}$, respectively, for the red- and blueshifted outflow lobes, and a total outflow mass of $\sim 140 \mathrm{M}_{\odot}$ at velocities $\gtrsim 2.5 \mathrm{~km} \mathrm{~s}^{-1}$ from the systemic velocity. The outflow mass is therefore comparable with the condensation mass. We list in Table 3 the derived outflow mass as well as its momentum $P=\sum m\left(v_{i}\right) v_{i}$, kinetic energy $E=\frac{1}{2} \sum m\left(v_{i}\right) v_{i}^{2}$, dynamical timescale $t_{d}$, mass-outflow rate $M / t_{d}$, momentum supply rate $P / t_{d}$, mechanical luminosity $E / t_{d}$, and collimation factor $q=D_{\max } / D_{\min }$ (where $D_{\max }$ and $D_{\min }$ correspond to the major and minor axis of the outflow). The dynamical timescale is defined as $r_{f} /\langle v\rangle$, where $\langle v\rangle$ is $P / M$ and $r_{f}$ is the outflow radius corresponding to half the separation between the centroids of red and blue lobes. Note that the collimation

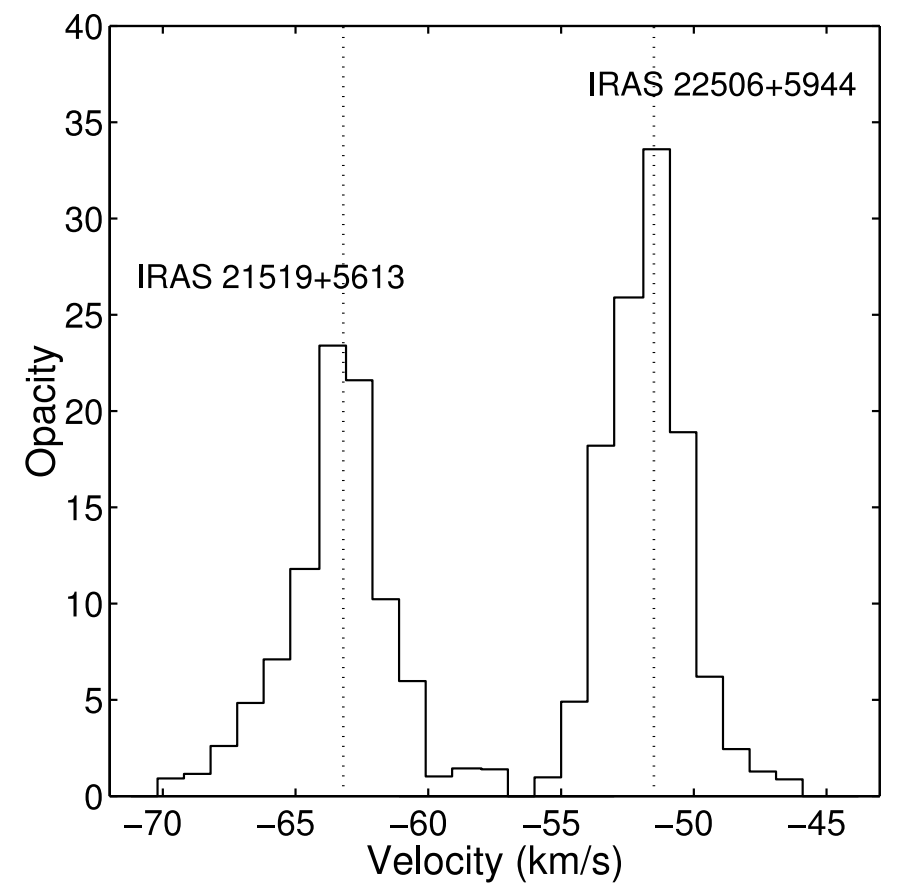

FIG. 8.-Derived opacity of the ${ }^{12} \mathrm{CO}$ emission as a function of velocity for IRAS $21519+5613$ and IRAS $22506+5944$. The dashed vertical lines show the $V_{\text {lsr }}$ of the individual sources. 
TABLE 3

Outflow Parameters

\begin{tabular}{|c|c|c|}
\hline Parameter & IRAS $21519+5613^{\mathrm{a}}$ & IRAS $22506+5944^{\mathrm{b}}$ \\
\hline Inclination angle (assumed) & 45 & 45 \\
\hline 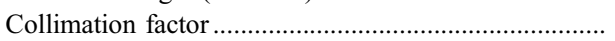 & $1.6 / 2.4^{\mathrm{c}}$ & $1.5 / 3.6^{\mathrm{c}}$ \\
\hline \multicolumn{3}{|l|}{ Outflow mass (derived from ${ }^{12} \mathrm{CO}$ ): } \\
\hline Redshifted $\left(M_{\odot}\right)$ & 62 & 13 \\
\hline Blueshifted $\left(M_{\odot}\right)$ & 80 & 7 \\
\hline Total $\left(M_{\odot}\right)$ & 140 & 20 \\
\hline Outflow radius $(\mathrm{pc})$ & 0.3 & 0.2 \\
\hline Mass-weighted mean velocity $\left(\mathrm{km} \mathrm{s}^{-1}\right) \ldots \ldots \ldots \ldots \ldots \ldots \ldots \ldots$ & 3.4 & 9.4 \\
\hline 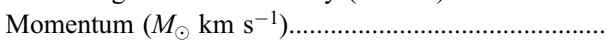 & 480 & 180 \\
\hline Kinetic energy (ergs) & $2.0 \times 10^{46}$ & $2.5 \times 10^{46}$ \\
\hline Dynamical timescale (yr) & 80,000 & 19,000 \\
\hline 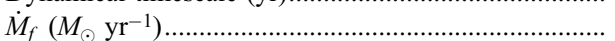 & $1.8 \times 10^{-3}$ & $1.1 \times 10^{-3}$ \\
\hline Momentum supply rate (force) $\left(M_{\odot} \mathrm{km} \mathrm{s}^{-1} \mathrm{yr}^{-1}\right) \ldots \ldots$ & $6.0 \times 10^{-3}$ & $9.5 \times 10^{-3}$ \\
\hline Mechanic luminosity $\left(L_{\odot}\right)$ & 2.1 & 11 \\
\hline
\end{tabular}

${ }^{\mathrm{a}}$ Redshifted emission integrated from -60.6 to $-46.4 \mathrm{~km}^{-1}$, corresponding to $2.6 \mathrm{~km} \mathrm{~s}^{-1} \lesssim v_{\text {flow }} \lesssim$ $16.8 \mathrm{~km} \mathrm{~s}^{-1}$, and blueshifted emission integrated from -78.9 to $-65.7 \mathrm{~km}^{-1}$, corresponding to $2.5 \mathrm{~km}$ $\mathrm{s}^{-1} \lesssim v_{\text {flow }} \lesssim 15.7 \mathrm{kms}^{-1}$.

${ }^{\mathrm{b}}$ Redshifted emission integrated from -47.4 to $-14.9 \mathrm{~km}^{-1}$, corresponding to $4.1 \mathrm{~km} \mathrm{~s}^{-1} \lesssim v_{\text {flow }} \lesssim$ $36.6 \mathrm{kms}^{-1}$, and blueshifted emission integrated from -107.3 to $-55.5 \mathrm{~km} \mathrm{~s}^{-1}$, corresponding to $4.0 \mathrm{~km}$ $\mathrm{s}^{-1} \lesssim v_{\text {flow }} \lesssim 55.8 \mathrm{~km} \mathrm{~s}^{-1}$.

${ }^{c}$ Deconvolution of the synthesized beam and then fitting with a two-dimensional Gaussian structure.

factors for the two outflows are calculated from the emission integrated over the outer line wings only as shown in the bottom panels of Figs. 4 and 7.

We infer a dynamical timescale for the outflow of $80,000 \mathrm{yr}$, with a corresponding mass-outflow rate of $1.8 \times 10^{-3} M_{\odot} \mathrm{yr}^{-1}$. By comparison, the ${ }^{12} \mathrm{CO}$ outflow masses associated with lowmass YSOs $\left(L_{\mathrm{bol}} \sim 1-10 L_{\odot}\right)$ typically range from 0.01 to a few $M_{\odot}$, with corresponding mass-outflow rates of $10^{-6}$ to $10^{-5} M_{\odot}$ $\mathrm{yr}^{-1}$ (e.g., Cabrit \& Bertout 1992). Note that the dynamical timescale and hence mass-outflow rate are computed assuming that the outflow axis is inclined by $45^{\circ}$. If the inclination angle is instead $15^{\circ} / 75^{\circ}$, then we would have under-/overestimated the dynamical timescale and over-/underestimated the mass-outflow rate by a factor of 4 . The collimation factor (ratio of major to minor axis of each outflow lobe) derived from the image of the outer line-wing emission is 1.6, comparable with those of known outflows associated with luminous YSOs (Richer et al. 2000). Because the outflow lobes are not well resolved along their minor axes, however, the derived collimation factor may be a lower limit. If the outflow lobes can be represented by two-dimensional Gaussian structures, then after deconvolution of the synthesized beam, we derive collimation factors of 2.1 for the blueshifted and 2.6 for the redshifted lobes.

\subsubsection{IRAS $22506+5944$}

The inferred physical parameters of the outflow associated with IRAS $22506+5944$ are listed also in Table 3. The derived molecular gas mass is 16 and $8 M_{\odot}$, respectively, at red- and blueshifted velocities larger than $\sim 4 \mathrm{~km} \mathrm{~s}^{-1}$ from the systemic velocity. Applying the same method as described above for removing the contribution from ambient gas, we find corresponding gas masses of 13 and $7 M_{\odot}$. The total outflow mass of $\sim 20 M_{\odot}$ is only one-seventh that associated with IRAS $21519+5613$, and an order of magnitude smaller than the mass of the molecular condensation. The dynamical timescale, again assuming an inclination angle of $45^{\circ}$, is 19,000 yr, a factor of $\sim 4$ smaller. Although IRAS
$22506+5944$ may therefore be at an earlier stage of evolution than IRAS $21519+5613$, it has a comparable mass-outflow rate of $1.1 \times 10^{-3} M_{\odot} \mathrm{yr}^{-1}$. The derived collimation factor of 1.5 is again likely to be a lower limit because the outflow lobes are not well resolved along their minor axes. Assuming as before that the outflow lobes can be represented by twodimensional Gaussian structures, the collimation factor is then 3.6 for both the blue- and redshifted lobes.

We emphasize that our above estimates of outflow masses do not include gas at very low velocities, where it is virtually impossible to separate the outflow from ambient gas (at $V_{\mathrm{lsr}} \pm$ $2.5 \mathrm{~km} \mathrm{~s}^{-1}$ for IRAS $21519+5613$ and $V_{\mathrm{lsr}} \pm 4 \mathrm{~km} \mathrm{~s}^{-1}$ for IRAS $22506+5944)$. This is a problem common to most, if not all, measurements of outflow masses in ${ }^{12} \mathrm{CO}$. Applying the method described above to estimate the contribution from ambient gas to the total emission at these very low velocities, we find an outflow mass of $\sim 290 M_{\odot}$ at $v_{\text {flow }} \lesssim 2.5 \mathrm{~km} \mathrm{~s}^{-1}$ for IRAS $22519+5613$ and $\sim 190 M_{\odot}$ at $v_{\text {flow }} \lesssim 4 \mathrm{~km} \mathrm{~s}^{-1}$ for IRAS $22506+5944$, larger than their individual outflow masses at higher velocities.

\subsection{Mass/Position versus Velocity}

\subsubsection{Mass-Velocity Diagram}

The outflows associated with low-mass YSOs usually show a power-law relation between mass and velocity, such that there is more mass at a lower velocity. That is, their outflows exhibit a "mass spectrum" $m(v) \propto v^{-\gamma}$, where the power index $\gamma$ is typically $\sim 1.8$. In some cases, the mass spectrum has a broken power law, with the slope steepening at outflow velocities $v_{\text {flow }} \gtrsim 10 \mathrm{~km} \mathrm{~s}^{-1}$, where $\gamma \approx 3-4$. From a compilation of 22 outflow sources with luminosities ranging from 1 to $3 \times 10^{5} L_{\odot}$, Richer et al. (2000) found that at low velocities $\gamma$ is similar in both low- and high-luminosity sources. At velocities above the breakpoint, however, $\gamma$ is 3-4 for lowmass YSOs but 3-8 for luminous YSOs. One possible explanation for the observed broken power-law relationship is to suppose that the low-velocity gas comprises ambient material entrained by the high-velocity gas (Bachiller \& Tafalla 1999). 

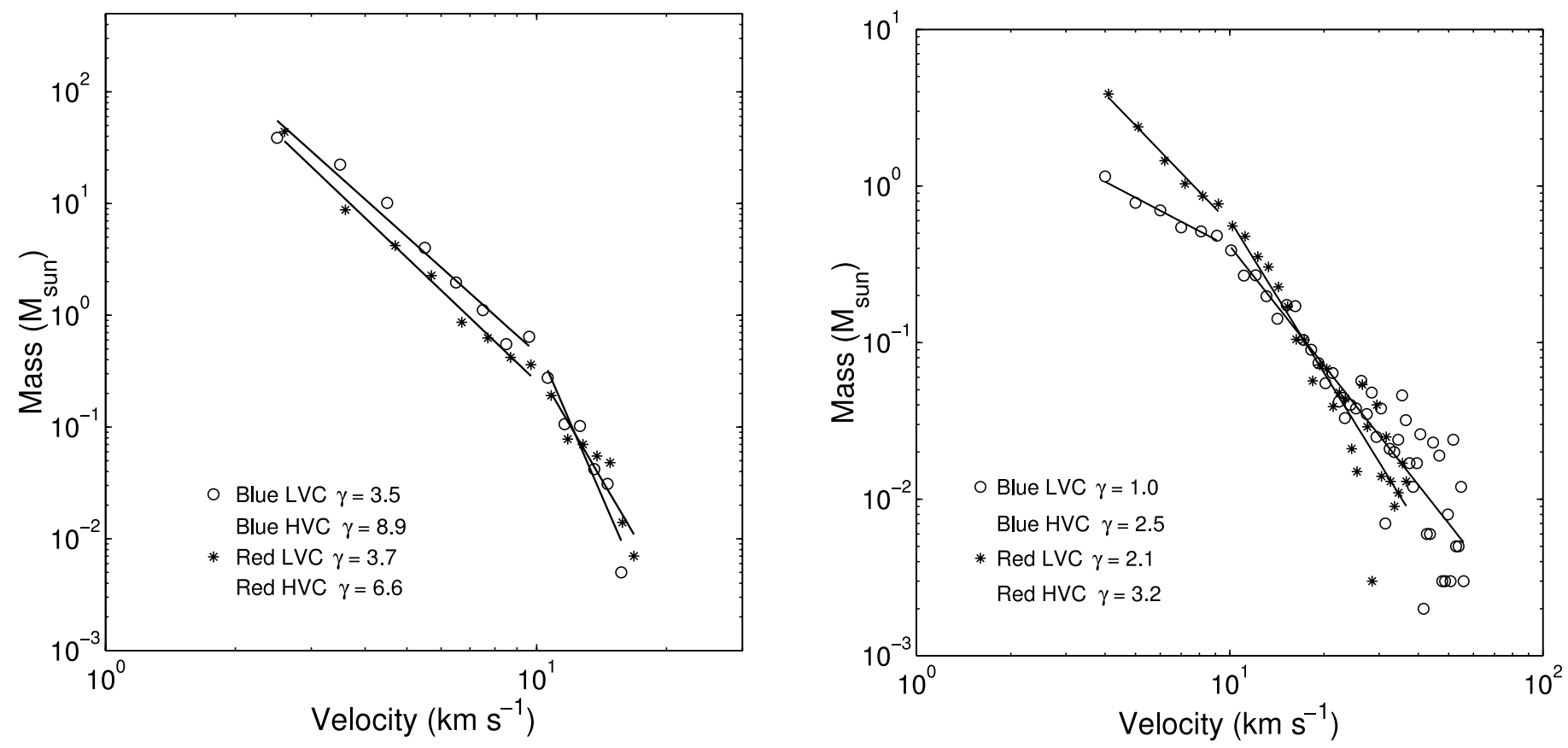

FIG. 9.-Outflow mass as a function of flow velocity for IRAS $21519+5613$ (left) and IRAS 22506+5944 (right), calculated for each channel of width $1.02 \mathrm{~km}$ $\mathrm{s}^{-1}$. In each panel, the stars and circles denote the red- and blueshifted emission, respectively. Power-law fits of the form of $m(v) \propto v^{-\gamma}$ are shown by solid lines, with the best-fit $\gamma$ indicated in the panel. LVC denotes the low-velocity component below the break point at $v_{\text {flow }} \approx 10 \mathrm{~km} \mathrm{~s}^{-1}$, and $\mathrm{HVC}$ denotes the high-velocity component above this break.

We consider this possibility for the outflows studied here in $\S 5.3 .1$.

The mass-velocity diagrams of the outflows from IRAS $21519+5613$ and IRAS $22506+5944$ are shown in Figure 9. For these outflows, we have used the methods described in $\S 4.2$ to correct for the opacity of ${ }^{12} \mathrm{CO}$ emission and confusion with ambient gas. The mass spectra of the individual outflows can be fitted by a broken power law with the slope steepening at $v_{\text {flow }} \gtrsim 10 \mathrm{~km} \mathrm{~s}^{-1}$ in both cases, as shown by the solid lines. The slopes at both low and high velocities, however, are steeper for IRAS $21519+5613$ than for IRAS 22506+5944. At low velocities $\left(v \lesssim 10 \mathrm{~km} \mathrm{~s}^{-1}\right), \gamma \approx 3.5$ for the blue and $\gamma \approx 3.7$ for the red lobes of IRAS $21519+5613$, but $\gamma \approx 1.0$ for the blue and $\gamma \approx 2.1$ for the red lobes of IRAS $22506+5944$. At higher velocities $\left(v \gtrsim 10 \mathrm{~km} \mathrm{~s}^{-1}\right), \gamma \approx 8.9$ for the blue and $\gamma \approx 6.6$ for the red lobes of IRAS $21519+5613$, but $\gamma \approx 2.5$ for the blue and $\gamma \approx 3.2$ for the red lobes of IRAS $22506+5944$. Note that the outflow velocities reported here are not corrected for inclination angle. Different inclination angles do not change the inferred values of $\gamma$ but may change the velocity at the break point.

\subsubsection{Position-Velocity Diagrams}

The position-velocity (P-V) diagrams of the two outflows measured along a line joining the centroids of their red and blue lobes are shown in Figure 10 . There is a spatially extended molecular gas component at $V_{\mathrm{lsr}} \approx-10 \mathrm{~km} \mathrm{~s}^{-1}$ in the direction of IRAS $22506+5944$ not associated with the outflow. In the P-V diagram of IRAS $22506+5944$, the velocity of the outflow can be seen to increase linearly with distance from the geometric center of both the blue- and redshifted lobes. This is a common feature of bipolar molecular outflows associated with both low- and high-luminosity YSOs, for example, Monoceros R2 (Meyers-Rice \& Lada 1991) and NGC 2264G (Lada \& Fich 1996). By contrast, the P-V diagram of
IRAS $21519+5613$ shows a decreasing maximum velocity with increasing radial distance. The implications of the kinematical feature are discussed in $\S 5.3 .1$.

\section{DISCUSSION}

\subsection{Outflow Properties}

\subsubsection{Comparison with Other Luminous YSOs}

Approximately 100 outflows apparently associated with luminous YSOs (with and without detectable UC-H II regions) have been reported (Shepherd \& Churchwell 1996; Churchwell 1999; Zhang et al. 2001; Beuther et al. 2002a). Their typical properties, as deduced from ${ }^{12} \mathrm{CO}$ observations, are the following: (1) masses in the range from tens to a few hundreds of $M_{\odot}$ (away from very low velocities where there is confusion with ambient gas); (2) ages (dynamical timescales) of $\sim 10^{4} \mathrm{yr}$ and mass-flow rates in the range $10^{-4}$ to $10^{-2} M_{\odot} \mathrm{yr}^{-1}$; (3) energetics and luminosities over an order of magnitude larger than outflows associated with low-mass YSOs. The derived outflow properties of IRAS $21519+5613$ and IRAS $22506+5944$ (i.e., mass of $\sim 100 M_{\odot}$ away from ambient gas velocities, massflow rate of $\sim 10^{-3} M_{\odot} \mathrm{yr}-1$, momentum of $\sim 100 M_{\odot} \mathrm{km}$ $\mathrm{s}^{-1}$, energy of $\sim 10^{46}$ ergs, and mechanical luminosities of $\sim 10 L_{\odot}$ ) are comparable with those of known outflows from luminous YSOs.

Where mapped at relative high angular resolutions of $\sim 15^{\prime \prime}-20^{\prime \prime}$, the outflows associated with high-luminosity YSOs appear to be less well collimated than those from lowluminosity YSOs (Richer et al. 2000 and references therein). The latter typically have collimation factors of $q \sim 2-3$, whereas luminous YSOs exhibit $q \sim 1-1.8$. An examination of published maps, however, suggests that outflows from luminous YSOs are usually not well (if at all) resolved along their minor axes, making many of the reported collimation factors likely lower limits. Furthermore, in some cases, the 

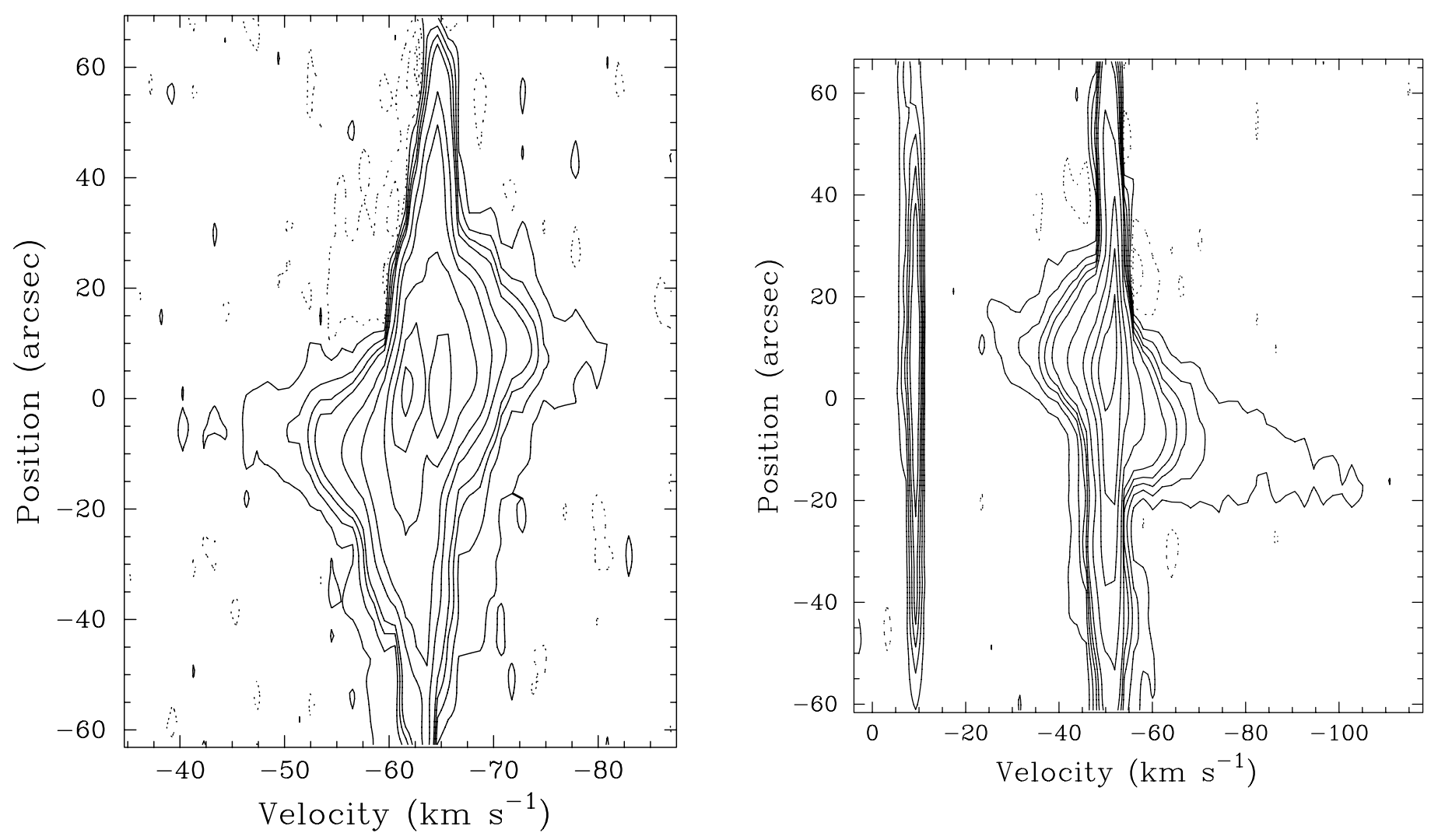

FIG. 10.- ${ }^{12} \mathrm{CO}$ P-V diagram of IRAS $21519+5613$ (left) and IRAS $22506+5944$ (right) along a line intersecting the centroids of their respective red- and blueshifted outflow lobes. For IRAS $22506+5944$, the molecular gas component at $V_{\mathrm{lsr}} \sim 10 \mathrm{~km} \mathrm{~s}^{-1}$ is unrelated to the outflow (see $\S 4.3 .2$ ).

outflow may be truncated at the base (e.g., G192.16-3.82; Shepherd et al. 1998), leading to an apparently small collimation factor. In this paper, we deduced the outflow collimation factors in two ways: by measuring the lobe dimensions directly from the derived images (similar to previous studies), and after deconvolving the synthesized beam assuming that the outflow lobes can be represented by two-dimensional Gaussian structures. With the first method, we derive collimation factors of $\sim 1.5$ for both cases, comparable with the above-mentioned published values. With the second method, we derive significantly higher collimation factors of $\sim 2.4$ for IRAS $21519+5613$ and $\sim 3.6$ for IRAS $22506+5944$. There are reports of even more highly collimated outflows from luminous YSOs; recently, Beuther et al. (2002a, 2002b) reported $q \sim 10$ for the outflow from the luminous $\left(\sim 10^{4} L_{\odot}\right)$ YSO IRAS $05358+3543$. Thus, the available evidence does not rule out the possibility that many outflows from luminous YSOs may be highly collimated and perhaps have comparable collimation factors to outflows from low-mass YSOs.

\subsubsection{Number of Outflows}

One of the primary challenges in studying molecular outflows associated with luminous YSOs is to positively identify the number of outflows present, as well as their individual driving sources. Because high-mass stars usually form in groups, it would hardly be surprising to find multiple molecular outflows associated with what appears to be a single massive star-forming condensation. Indeed, several such condensations exhibiting more than one molecular outflow have been reported (e.g., Beuther et al. 2002a, 2002b). The observations presented here with a spatial resolution of $\sim 0.3 \mathrm{pc}$ show evidence for only a single bipolar outflow originating from a compact dust and gas condensation in the cases of both IRAS $21519+5613$ and IRAS $22506+5944$. The relatively high collimation factors of $2-3$, comparable with those of low-mass YSOs, further argue for a single outflow in each case. Furthermore, we have the sensitivity to detect any outflows from low-mass YSOs that are not spatially aligned with the observed outflows. Nevertheless, it is always possible that observations at high angular resolutions may reveal that what appears to be a single collimated outflow is actually composed of several closely aligned outflows. Indeed, observations at high spatial resolutions toward several nearby clustered star formation regions (e.g., IRAS 19410+2336 and W75 N) reveal multiple outflows within a scale of $0.3 \mathrm{pc}$ (Beuther, Schilke, \& Stanke 2003; Shepherd, Testi, \& Stark 2003).

\subsection{3. $\dot{M}_{f}$ versus $L_{\mathrm{bol}}$ Relation}

The CO-inferred mass-outflow rate of YSOs appears to increase with bolometric luminosity over the entire range measured thus far from $\sim 1$ to $\sim 10^{6} L_{\odot}$ (Shepherd \& Churchwell 1996; Churchwell 2002). When placed on this $\dot{M}_{f}$ versus $L_{\mathrm{bol}}$ diagram, neglecting (as in previous studies) the outflow mass at very low velocities confused with ambient gas, the two outflows studied here also fall on this relationship within (the quite large) observational scatter. Although this relationship has been used to argue for a common outflowdriving mechanism for all sources, the physical significance of this relationship remains uncertain (for a brief review, see Churchwell 2002). More recent studies have called into question the existence of this relationship (Beuther et al. 2002a). We add that until the outflowing gas at very low velocities - which may constitute a significant fraction if not the 
bulk of the $\mathrm{CO}$ outflow-is properly taken into account, the functional form of this dependence, even if factual, must be treated with caution.

\subsection{Outflow Driving Sources}

\subsubsection{Massive Protostar}

The large outflow mass and high mass-outflow rate, together with the high IRAS bolometric luminosities of $\sim 2 \times 10^{4} L_{\odot}$, suggest that both outflows are driven by massive stars. Furthermore, the short outflow dynamical timescale of $\lesssim 10^{5} \mathrm{yr}$ and lack of a detectable UC-H II region suggest that the driving source may be a protostar. That is, for the free-free emission from any $\mathrm{UC}-\mathrm{H}$ II region to be $\lesssim 0.3 \mathrm{mJy}$ at $6 \mathrm{~cm}$ as observed, the $\operatorname{Ly} \alpha$ flux from the central source must be $\lesssim 10^{45} \mathrm{~s}^{-1}$ if the region is optically thin but may be somewhat higher if it is optically thick. A main-sequence star with the measured bolometric luminosity, a B0 star, has a Ly $\alpha$ flux of $\sim 2 \times 10^{47} \mathrm{~s}^{-1}$ that would be sufficient to excite a detectable $\mathrm{UC}-\mathrm{H}$ II region.

If the sources studied here are indeed protostars that owe their luminosity primarily to accretion, their mass-accretion rate $\dot{M} \approx 6 \times 10^{-4} R_{*} / M_{*} M_{\odot} \mathrm{yr}^{-1}$, where $R_{*}$ and $M_{*}$ are the protostellar radius and mass normalized to the Sun. Although $R_{*}$ and $M_{*}$ are not known, a protostar with a given $M_{*}$ is expected to have a larger $R_{*}$ than its corresponding value on the main sequence, where $1 \gtrsim R_{*} / M_{*} \gtrsim 0.25$ for $0.1 M_{\odot} \lesssim$ $M_{*} \lesssim 60 M_{\odot}$ (Schmidt-Kaler 1982). The inferred massaccretion rate of $\gtrsim 10^{-4} M_{\odot} \mathrm{yr}^{-1}$ is more than sufficient (by factors of 10-100) to quench an UC-H II region around a main-sequence (B0) star of the observed luminosity (Walmsley 1995), let alone a larger and therefore cooler protostar. Note that the above argument can be applied to any protostar whose luminosity exceeds $\sim 10^{3} L_{\odot}$ so long as its primary energy source is accretion; in such a case, the mass-accretion rate needed to produce the observed luminosity is easily sufficient to quench an $\mathrm{UC}-\mathrm{H}$ II region.

Both IRAS $21519+5613$ and IRAS 22506+5944 exhibit bright water masers (Wouterloot et al. 1993; Migenes et al. 1999) coincident with the centroids of their dust and gas condensations identified in our observations. This bolsters the case that both outflow driving sources are relatively young massive stars. Most bright water masers found in star-forming regions are associated with sources having far-IR luminosities $\gtrsim 10^{4} L_{\odot}$ (Palagi et al. 1993). Furthermore, interferometric observations show that these water masers are more commonly associated with hot molecular cores, and therefore possible protostars, rather than $\mathrm{UC}-\mathrm{H}$ II regions, and therefore main-sequence stars (Kurtz et al. 2000 and references therein).

\subsubsection{Non- or Weakly Ionizing Main-Sequence Star}

The above arguments assume that a single star is responsible for producing all the observed luminosity. It is well known, however, that massive stars often form in groups with other massive as well as an even greater number of lower mass stars. If a part of the observed bolometric luminosity originates from stars with no appreciable ionizing flux, then it may be that the dominant star, even if it is on the main sequence, is too cool to excite a detectable UC-H II region. Indeed, in the case of IRAS 21519+5613, Molinari et al. (2002) detected two dust components at $3 \mathrm{~mm}$ with the minor component having about half the flux density of the main component (which we identify with the driving source).
For the two objects studied here not to produce a detectable $\mathrm{UC}-\mathrm{H}$ II region that is optically thin at $6 \mathrm{~cm}$, the most luminous star must have a spectral type of B2 or later, corresponding to a bolometric luminosity $\$ 3000 L_{\odot}$. If the $\mathrm{UC}-\mathrm{H}$ II region is optically thick, then the star can have an even earlier spectral type; for example, if the emission measure is $\sim 10^{9} \mathrm{pc} \mathrm{cm}^{-6}$ ( 2 orders of magnitude higher than is commonly exhibited by $\mathrm{UC}-\mathrm{H}$ II regions), the central star can be as early as $\mathrm{B} 0.5$, with $L_{\mathrm{bol}} \sim 1 \times 10^{4} L_{\odot}$. Any continuing accretion would further suppress an $\mathrm{UC}-\mathrm{H}$ II region; an ionized accretion flow has been identified in the $\mathrm{UC}-\mathrm{H}$ II region G10.6-0.4 (Keto 2002), indicating that accretion can continue during this phase of evolution. We note that a driving source with a fraction of the observed IRAS luminosity would not violate the $\dot{M}_{f}$ versus $L_{\mathrm{bol}}$ relationship of Shepherd \& Churchwell (1996) given the considerable scatter in this relationship. High angular resolution observations of the dust to determine the number of luminous sources, as well as deep searches for any free-free emission associated with the driving source, are therefore important for distinguishing between massive protostars and main-sequence stars.

\subsubsection{Implications for Star Formation Models}

The inferred dynamic timescale for the outflows of $10^{4}$ $10^{5} \mathrm{yr}$ is comparable with the Kelvin-Helmholtz contraction timescale for a star with mass of $\sim 10 M_{\odot}$. If the two driving sources are therefore early-B main-sequence stars, the time-averaged mass-accretion rate needed to form these stars is $10^{-3}$ to $10^{-4} M_{\odot} \mathrm{yr}^{-1}$. On the other hand, if the two objects are protostars, then their masses cannot be very much larger than $\sim 10 M_{\odot}$, otherwise the central object would already have contracted onto the main sequence. In such a case, as shown above, their present mass-accretion rate must be $\gtrsim 10^{-4} M_{\odot} \mathrm{yr}^{-1}$.

Thus, no matter the evolutionary state of the star, the implied mass-accretion rate is much higher than can be accommodated by simple inside-out collapse models, even at temperatures relevant for hot molecular cores (Cesaroni et al. 1994). In these models, the predicted mass-accretion rates range from $4 \times$ $10^{-6}(T \approx 20 \mathrm{~K})$ to $10^{-4} M_{\odot} \mathrm{yr}^{-1}(T \approx 200 \mathrm{~K})$. For these models to remain relevant, one would then have to appeal to conditions that can significantly increase the mass-accretion rate, such as that proposed by McKee \& Tan (2003). In the latter model, the implied high inward pressures can overcome the increasingly stronger outward radiation pressure from the accretion surface as the central protostar gains mass. This problem could be further alleviated if accretion occurs primarily through a disk, as considered by Jijina \& Adams (1996). In such a case, however, sufficient ionizing photons may be able to escape along the polar directions to generate a detectable $\mathrm{UC}-\mathrm{H}$ II region (if the accretion surface is sufficiently hot).

\subsection{Outflow Driving Agents}

The origin of the large outflow masses associated with luminous YSOs is poorly understood. In low-mass YSOs, the bulk of the molecular outflow observed in $\mathrm{CO}$ is thought to comprise swept-up ambient molecular gas. It is not clear, however, whether entrainment alone can produce the large outflow masses associated with luminous YSOs (Churchwell 1997; Churchwell 2002). Here we consider this issue from a kinematic and energetic viewpoint. If the observed $\mathrm{CO}$ outflow comprises predominantly entrained material, the driving 
agent must have significantly higher velocities and may have lower mass. The composition of this high-velocity component is poorly understood (Bachiller \& Tafalla 1999), but the most obvious possible forms are ionized gas, molecular gas, and/or atomic gas. Below we discuss the two possibilities addressed by our observations.

\subsection{1. "High-Velocity" Molecular Gas}

As shown in $\S 4.3 .1$, the mass-velocity diagrams of the two outflows can be characterized by a broken power law with the spectrum steepening at velocities $\gtrsim 10 \mathrm{~km} \mathrm{~s}^{-1}$. One possible explanation for this behavior is that the high-velocity component (HVC) at $v_{\text {flow }} \gtrsim 10 \mathrm{~km} \mathrm{~s}^{-1}$ is the actual driving agent and the low-velocity component (LVC) at $v_{\text {flow }} \lesssim 10 \mathrm{~km} \mathrm{~s}^{-1}$ comprises entrained ambient gas (for a recent review, see Bachiller \& Tafalla 1999). If this is the case, the HVC should have enough thrust (i.e., momentum supply rate) to drive the LVC and show evidence of injecting momentum into this gas. For IRAS $21519+5613$ and IRAS $22506+5944$, the ratio of momentum supply rates in the LVC and HVC, neglecting the very low velocity flow confused with the ambient gas, is approximately 40 and 1 respectively. By also including this very low velocity outflowing gas as estimated in $\S 4.2 .2$, the corresponding values become 60 for IRAS $21519+5613$ and 3 for IRAS $22506+5944$.

If the HVC is indeed injecting momentum into the LVC, the former should decelerate and hence its P-V diagram show a decreasing velocity with increasing distance from the outflow origin. Such a relationship has been found in the HVCs of the low-mass systems L1448 and IRAS $03282+3035$, where the $\mathrm{HVC}$ takes the form of discrete condensations referred to as molecular bullets (Bachiller et al. 1990; Bachiller, MartínPintado, \& Planesas 1991). In the case of IRAS 21519+5613, the P-V diagram of the HVC, especially in the redshifted lobe, shows such a signature. In the case of IRAS $22506+5944$, however, no such signature is seen. It is possible that some of the high-velocity bullets may have recently been ejected from the central YSO and have not yet interacted with the ambient gas. In such a case, the HVC should show two different kinematic structures: bullets with a range of velocities close to the source that have yet to interact with the ambient gas and more slowly moving bullets farther away that have entrained ambient gas. No such kinematic signatures can be discerned from the P-V diagram of IRAS $22506+5944$.

In summary, the HVC of IRAS $22506+5944$ does not appear to have enough thrust to drive the bulk of the LVC and, furthermore, does not exhibit the expected kinematic signature of decelerating gas. In the case of IRAS $21519+5613$, the HVC exhibits the expected kinematic signature but does not have enough thrust. Note, however, that we have implicitly assumed that both the HVC and LVC have the same kinetic temperatures; it is entirely possible that the HVC is more highly excited, so that its mass and momentum supply rate is significantly higher than the values we have derived. Observations of the outflow at higher transitions of $\mathrm{CO}$ are required to test this possibility.

\subsubsection{Ionized Jets}

At the extreme, the high-velocity flow may be predominated ionized. When observed at sufficiently high angular resolutions, many ionized source associated with mostly lowluminosity YSOs have been found to be elongated along the direction of their associated molecular outflows (Anglada
1996). The momentum supply rates of such ionized jets, however, are generally 1-2 orders of magnitude lower than that of molecular outflows and therefore cannot be the primary driving agent. This appears to be true not only for low-mass cases, but also for luminous YSOs (Gómez et al. 1999; Hofner et al. 1999; Shepherd \& Kurtz 1999). Here we examine the specific cases of IRAS $21519+5613$ and IRAS $22506+5944$.

Recall that a collimated wind produces a higher radio flux density than a spherically symmetric wind with otherwise similar physical properties (Reynolds 1986). With an opening angle of $\theta_{0}$ (in radians), inclination $i$, mass-loss rate $\dot{M}_{f}$, and velocity $v_{\text {wind, }}$, the flux density of an ionized flow at a given observing frequency $\nu$ is given by

$$
\begin{aligned}
& S_{\nu}(\mathrm{mJy}) \approx 40 \theta_{0}^{-4 / 3}(\sin i)^{1 / 3}\left(\frac{\nu}{10 \mathrm{GHz}}\right)^{0.6}\left(\frac{T_{e}}{10^{4} \mathrm{~K}}\right)^{0.1} \\
& \times\left(\frac{\dot{M}_{f}}{10^{-5} M_{\odot} \mathrm{yr}^{-1}}\right)^{4 / 3}\left(\frac{v_{\text {wind }}}{1000 \mathrm{~km} \mathrm{~s}^{-1}}\right)^{-4 / 3}\left(\frac{d}{\mathrm{kpc}}\right)^{-2},
\end{aligned}
$$

(Panagia \& Felli 1975; Reynolds 1986). An outflow collimation factor of $\sim 2-3$ for IRAS $21519+5613$ and IRAS $22506+5944$ implies that any ionized jets driving their molecular outflows have opening angles $\lesssim 1$ radian.

The largest uncertainty in estimating the mass-loss rate of ionized jets from radio continuum measurements alone is the jet velocity. The ionized jets in low-mass YSOs have a velocity of $\sim 100 \mathrm{~km} \mathrm{~s}^{-1}$, comparable with the Keplerian velocity at a few solar radii where the jet may be launched (Shu et al. 2000). The Herbig-Haro objects $\mathrm{HH} 80 / 81$, associated with a luminous YSO with $L_{\text {bol }}$ of $2 \times 10^{4} L_{\odot}$, have measured radial velocities of $700 \mathrm{~km} \mathrm{~s}^{-1}$ (Heathcote, Reipurth, \& Raga 1998). This corresponds to be a Keplerian velocity at a distance of a few solar radii from a central object with mass of $\sim 10 M_{\odot}$. Assuming $v_{\text {wind }} \approx 700 \mathrm{~km} \mathrm{~s}^{-1}, \theta_{0} \approx 1, T_{e} \approx$ $10^{4} \mathrm{~K}$, and $i \approx 45^{\circ}$, the measured upper limit of $0.3 \mathrm{mJy}$ at $6 \mathrm{~cm}$ $(\nu=5 \mathrm{GHz})$ for sources at a distance $\sim 5 \mathrm{kpc}$ implies an upper limit to the mass-outflow rate of $\sim 10^{-6} M_{\odot} \mathrm{yr}^{-1}$ and the corresponding momentum supply rate of $\sim 10^{-3} M_{\odot} \mathrm{yr}^{-1} \mathrm{~km}$ $\mathrm{s}^{-1}$. The latter is approximately 1 order of magnitude smaller than that inferred for the $\mathrm{CO}$ outflow.

\section{SUMMARY}

We have imaged at relatively high angular resolutions $\left(\sim 10^{\prime \prime}\right)$ the bipolar molecular outflows associated with the luminous far-IR sources IRAS $21519+5613$ and IRAS $22506+5944$, as well as their associated molecular gas and dust condensations. Our main results can be summarized as follows.

1. We resolved the outflows along their major axes and confirmed that both are aligned in approximately the east-west direction. In ${ }^{12} \mathrm{CO}(J=1-0)$, the outflow from IRAS $21519+5613$ was traced to a maximum velocity of $\sim 15 \mathrm{~km} \mathrm{~s}^{-1}$, whereas that from IRAS $22506+5944$ was traced to $\sim 50 \mathrm{~km}$ $\mathrm{s}^{-1}$. The derived outflow properties (i.e., mass of $\sim 100 M_{\odot}$, mass-flow rate of $\sim 10^{-3} M_{\odot} \mathrm{yr}^{-1}$, momentum of $\sim 100 M_{\odot} \mathrm{km}$ $\mathrm{s}^{-1}$, energy of $\sim 10^{46}$ ergs, and mechanical luminosities of $\sim 10 L_{\odot}$ ) are comparable with those of known outflows from luminous YSOs.

2. The outflows may be quite highly collimated: assuming that the outflow lobes can be represented by two-dimensional Gaussian structures, we infer collimation factors $q$ in the range 2.4-3.6. Such collimation factors are comparable with those 
exhibited by low-mass YSOs and add further evidence that massive outflows associated with luminous YSOs can be highly collimated.

3. Each outflow is centered on a dust and molecular gas condensation detected in the $3 \mathrm{~mm}$ continuum as well as ${ }^{13} \mathrm{CO}$ $(J=1-0)$ and $\mathrm{C}^{18} \mathrm{O}(J=1-0)$. These condensations have a mass of $200-300 M_{\odot}$ and a size $\lesssim 0.4 \mathrm{pc}$, comparable with those associated with other luminous YSOs (Cesaroni et al. 1997; Shepherd \& Kurtz 1999; Molinari et al. 2002).

4. Both outflows show a break in their mass-velocity diagrams at a velocity of $\sim 10 \mathrm{~km} \mathrm{~s}^{-1}$, as is exhibited by a number of other massive outflows associated with luminous YSOs. We considered whether the high-velocity component $\left(\gtrsim 10 \mathrm{~km} \mathrm{~s}^{-1}\right)$ may drive the low-velocity component $\left(\leqslant 10 \mathrm{~km} \mathrm{~s}^{-1}\right)$ but found that the high-velocity component has neither a sufficient moment supply rate, nor, for IRAS $22506+5944$, the expected kinematics. Observations in higher transitions of ${ }^{12} \mathrm{CO}$ are necessary to determine whether the high-velocity component is more highly excited and hence its thrust underestimated.

5. As for other outflows, the molecular outflows of IRAS $21519+5613$ and IRAS $22506+5944$ cannot be driven primarily by a collimated ionized outflow. If not also molecular gas, the only remaining candidate is atomic hydrogen gas, as has been proposed and detected in some outflows associated with low-mass protostars (Giovanardi et al. 2000).

6. The large outflow mass, high mass-outflow rate, and short outflow dynamical timescale $\left(10^{4}-10^{5} \mathrm{yr}\right)$ add further evidence that both IRAS $21519+5613$ and IRAS $22506+5944$ are high-mass protostars. The inferred mass-accretion rate is more than sufficient to quench the development of an UC- $\mathrm{H}$ II region.

7. It is, however, entirely possible that the outflow driving source is an early- to mid-B main-sequence star that is the dominant member of a group/cluster (and hence contributes only a part of the observed bolometric luminosity) whose members do not produce sufficient ionizing photons to excite a detectable UC-H II region. Such a possibility is difficult to rule out for sources with bolometric luminosities close to $\sim 10^{4} L_{\odot}$, such as those observed here, and for many other claimed massive protostars. Observations of the dust emission at high angular resolutions are critical for a proper census of the number and nature of the exciting stars.

8. Whether protostars or main-sequence stars, we infer a mass accretion rate of $\gtrsim 10^{-4} M_{\odot} \mathrm{yr}^{-1}$ for both IRAS $21519+5613$ and IRAS $22506+5944$. Such mass-accretion rates are much higher than those predicted by simple inside-out collapse models but may be possible in variants such as that proposed by McKee \& Tan (2003).

We acknowledge valuable comments from an anonymous referee. We would like to thank the operators of the NRAO $12 \mathrm{~m}$ telescope and the BIMA array for their assistance during the observations. We also thank the National Science Council of Taiwan for support of this work through research grant NSC 92-2112-M-001-032 to J. Lim and a graduate student fellowship to Y.-N. Su.
Anglada, G. 1996, in ASP Conf. Ser. 93, Radio Emission from the Stars and the Sun, ed. A. R. Taylor \& J. M. Paredes (San Francisco: ASP), 3

Bachiller, R., Cernicharo, J., Martín-Pintado, J., Tafalla, M., \& Lazareff, B. 1990, A\&A, 231, 174

Bachiller, R., Martín-Pintado, J., \& Planesas, P. 1991, A\&A, 251, 639

Bachiller, R., \& Tafalla M. 1999, in The Origin of Stars and Planetary Systems, ed. C. J. Lada \& N. D. Kylafis (NATO ASI Ser. C, 540; Dordrecht: Kluwer), 227

Beuther, H., Schilke, P., Gueth, F., McCaughrean, M., Andersen, M., Sridharan, T. K., and Menten, K. M. 2002a, A\&A, 387, 931

Beuther, H., Schilke, P., Sridharan, T. K., Menten, K. M., Wamsley, C. M., \& Wyrowski, F. 2002b, A\&A, 383, 892

Beuther, H., Schilke, P., \& Stanke, T. 2003, A\&A, 408, 601

Bonnell, I. A., Bate, M. R., \& Zinnecker, H. 1998, MNRAS, 298, 93

Bronfman, L., Nyman, L.-Å., \& May, J. 1996, A\&AS, 115, 81

Cabrit, S., \& Bertout, C. 1992, A\&A, 261, 274

Cesaroni, R., Churchwell, E., Hofner, P., Walmsley, C. M., \& Kurtz, S. 1994, A\&A, 288, 903

Cesaroni, R., Felli, M., Testi, L., Walmsley, C. M., \& Olmi, L. 1997, A\&A, 325,725

Churchwell, E. 1997, ApJ, 479, L59

. 1999, in The Origin of Stars and Planetary Systems, ed. C. J. Lada \&

N. D. Kylafis (NATO ASI Ser. C, 540; Dordrecht: Kluwer), 515 2002, ARA\&A, 40, 27

Davis, C. J., Moriarty-Schieven, G., Eislöffel, J., Hoare, M. G., \& Ray, T. P. 1998, AJ, 115, 1118

Giovanardi, C., Rodríguez, L. F., Lizano, S., \& Cantó, J. 2000, ApJ, 538, 728 Gómez, J. F., Sargent, A. I., Torrelles, J. M., Ho, P. T. P., Rodríguez, L. F., Cantó, J., \& Garay, G. 1999, ApJ, 514, 287

Heathcote, S., Reipurth, B., \& Raga, A.C. 1998, AJ, 116, 1940

Hildebrand, R. H. 1983, QJRAS, 24, 267

Hofner, P., Cesaroni, R., Rodríguez, L. F., \& Martí, J. 1999, A\&A, 345, L43

Jijina, J., \& Adams, F. C. 1996, ApJ, 462, 874

Keto, E. 2002, ApJ, 568, 754

Kramer, C., Alves, J., Lada, C., Lada, E., Sievers, A., Ungerechts, H., \& Walmsley, M. 1998, A\&A, 329, L33

Kurtz, S., Cesaroni, R., Churchwell, E., Hofner, P., and Walmsley, C. M. 2000, in Protostars and Planets IV, ed. V. Mannings, A. P. Boss, \& S. S. Russell, S. S. (Tucson: Univ. Arizonia Press), 299

Lada, C. J., \& Fich M. 1996, ApJ, 459, 638
Masson, C. R., \& Chernin, L. M. 1993, ApJ, 414, 230

McKee, C. F., \& Tan, J. C. 2003, ApJ, 585, 850

Meyers-Rice, B. A., \& Lada, C. J. 1991, ApJ, 368, 445

Migenes, V., et al. 1999, ApJS, 123, 487

Molinari, S., Brand, J., Cesaroni, R., \& Palla, F. 1996, A\&A, 308, 573

Molinari, S., Brand, J., Cesaroni, R., Palla, F., \& Palumbo, G. G. C. 1998, A\&A, 336, 339

Molinari, S., Testi, L., Rodríguez, L. F., \& Zhang, Q. 2002, ApJ, 570, 758

Palagi, F., Cesaroni, R., Comoretto, G., Felli, M., \& Natale, V. 1993, A\&AS, 101,153

Panagia, N., \& Felli, M. 1975, A\&A, 39, 1

Raga, A., \& Cabrit, S. 1993, A\&A, 278, 267

Reynolds, S. P. 1986, ApJ, 304, 713

Richer, J. S., Shepherd, D. S., Cabrit, S., Bachiller, R., \& Churchwell, E. 2000 , in Protostars and Planets IV, ed. V. Mannings, A. P. Boss, \& S. S. Russell, (Tucson: Univ. Arizona Press), 867

Schmidt-Kaler, T. 1982, in Stars and Star Clusters, ed. K. Schaifers \& H. H. Voigt (Berlin: Springer), 1

Scoville, N. Z., Sargent, A. I., Sanders, D. B., Claussen, M. J., Masson, C. R., Lo, K. Y., \& Phillips, T. G. 1986, ApJ, 303, 416

Shepherd, D. S., \& Churchwell, E. 1996, ApJ, 472, 225

Shepherd, D. S., \& Kurtz, S. E. 1999, ApJ, 523, 690

Shepherd, D. S., Testi, L., \& Stark, D. P. 2003, ApJ, 584, 882

Shepherd, D. S., \& Watson, A. M. 2002, ApJ, 566, 966

Shepherd, D. S., Watson, A. M., Sargent, A. I., \& Churchwell, E. 1998, ApJ, 507,861

Shu, F. H., Najita, J. R., Shang, H., \& Li, Z.-Y. 2000, in Protostars and Planets IV, ed. V. Mannings, A. P. Boss, \& S. S. Russell, (Tucson: Univ. Arizona Press), 789

Shu, F. H., Ruden, S. P., Lada, C. J., \& Lizano, S. 1991, ApJ, 370, L31

Stahler, S. W., Palla, F., \& Ho, P. T. P. 2000, in Protostars and Planets IV, ed. V. Mannings, A. P. Boss, \& S. S. Russell, (Tucson: Univ. Arizona Press), 327

Vogel, S. N., Wright, M. C. H., Plambeck, R. L., \& Welch, W. J. 1984, ApJ, 283,655

Walmsley, M. 1995, Rev. Mexicana Astron. Astrofis. Ser. Conf., 1, 137

Wilson, T. L., \& Rood, R. T. 1994, ARA\&A, 32, 191

Wouterloot, J. G. A., Brand, J., \& Fiegle, K. 1993, A\&AS, 98, 589

Zhang, Q., Hunter, T. R., Brand, J., Sridharan, T. K., Molinari, S., Kramer, M. A., \& Cesaroni, R. 2001, ApJ, 552, L167 